\title{
Hybrid Digital-Analog Coding with Bandwidth Compression for Gaussian Source-Channel Pairs
}

\author{
Yadong Wang, Fady Alajaji, Senior Member, IEEE, and Tamás Linder, Senior Member, IEEE
}

\begin{abstract}
Three hybrid digital-analog (HDA) systems, denoted by HDA-I, HDA* and HDA-II, for the coding of a memoryless discrete-time Gaussian source over a discrete-time additive memoryless Gaussian channel under bandwidth compression are studied. The systems employ simple linear coding in their analog component and superimpose their analog and digital signals before channel transmission. Information-theoretic upper bounds on the asymptotically optimal mean squared error distortion of the systems are obtained under both matched and mismatched channel conditions. Allocation schemes for distributing the channel input power between the analog and the digital signals are also examined. It is shown that systems HDA* and HDA-II can asymptotically achieve the optimal Shannon-limit performance under matched channel conditions. Low-complexity and lowdelay versions of systems HDA-I and HDA-II are next designed and implemented without the use of error correcting codes. The parameters of these HDA systems, which employ vector quantization in conjunction with binary phase-shift keying modulation in their digital part, are optimized via an iterative algorithm similar to the design algorithm for channel-optimized vector quantizers. Both systems have low complexity and low delay, and guarantee graceful performance improvements for high CSNRs. For memoryless Gaussian sources the designed HDAII system is shown to be superior to the HDA-I designed system. When applied to a Gauss-Markov source under Karhunen-Loeve processing, the HDA-I system is shown to provide considerably better performance.
\end{abstract}

Index Terms-Broadcasting, hybrid digital-analog coding, joint source-channel coding, linear analog coding, robustness, vector quantization.

\section{INTRODUCTION}

W E consider the problem of transmitting a discrete-time analog-valued source over a discrete-time memoryless channel. Due to the often lacking channel information at the transmitter, a robust system is desirable for a wide range of channel conditions. In terms of the used modulation techniques, systems can be generally categorized as analog, digital or hybrid digital-analog (HDA).

One of the main advantages of digital communication systems is that they can be designed to (asymptotically) achieve

Paper approved by K. Rose, the Editor for Source/Channel Coding of the IEEE Communications Society. Manuscript received April 30, 2007; revised March 17, 2008 and September 6, 2008.

Parts of the material in this paper were presented at the 2005 IEEE Data Compression Conference (DCC'05), Snowbird, UT, March 2005 and the IEEE 23nd Biennial Symposium on Communications (23rd QBSC), Queen's University, Kingston, ON, Canada, May-June 2006. This work was supported in part by NSERC of Canada and PREA of Ontario.

Y. Wang is with the Bank of Montreal, 8th Floor, 302 Bay St., Toronto, Canada (e-mail: y.d.wang99@gmail.com)

F. Alajaji and T. Linder are with the Department of Mathematics and Statistics, Queen's University, Kingston, Ontario, Canada, K7L 3N6 (e-mail: \{fady, linder\}@mast.queensu.ca).

Digital Object Identifier 10.1109/TCOMM.2009.04.070165 the theoretical optimal performance for a fixed channel signalto-noise ratio (CSNR) via the separate design of optimal source and channel codes [27], [4]. Systems designed based on this principle are often referred to as tandem sourcechannel coding systems. There are, however, two fundamental disadvantages associated with digital tandem systems. One is the threshold effect: the system typically performs well at the design CSNR, while its performance degrades drastically when the true CSNR falls below the design CSNR. This effect is due to the quantizer's sensitivity to channel errors and the eventual breakdown of the employed error correcting code at low CSNRs (no matter how powerful it is). The other trait is the leveling-off effect: as the CSNR increases, the performance remains constant beyond a certain threshold. This is due to the non-recoverable distortion introduced by the quantizer which limits the system performance at high CSNRs.

The threshold effect can be partly remedied via digital joint source-channel coding (JSCC). By jointly designing the source and channel codes, many results (e.g., [9], [17]) show that noticeable gain can be obtained in terms of coding efficiency, reconstructed signal quality, coding delay and complexity. In particular, JSCC schemes are more robust than tandem systems at low CSNRs. However, such JSCC systems still suffer from the leveling-off effect at high CSNRs, since being digital systems, they employ quantization to "digitize" the source. On the other hand, the leveling-off effect is not a problem for analog systems (we call a discrete-time system analog if it uses an analog modulation technique such as amplitude modulation); actually, their performance can strictly increase as the CSNR increases. However, it is usually hard to incorporate efficient signal compression schemes in analog systems, particularly when channel bandwidth change is required and/or the source has memory.

Schemes that exploit the advantage of analog systems are studied by Ramstad and his co-authors in [16], [10], [6], [5], and [11]. These are based on the so-called direct source-channel mapping technique: the output of a source scalar/vector quantizer is mapped directly to a channel symbol using analog (or nearly analog) modulation, i.e., amplitude modulation or $M$-ary quadrature amplitude modulation (QAM) with $M \gg 1$. The direct source-channel codes also enjoy graceful degradation performance at low CSNRs. In [16], a robust image coding system is presented which combines subband coding and QAM. This system allows various compression levels based on block-wise classification. An improved image coding system is proposed in [6]; it utilizes both bandwidth compression and bandwidth expansion mappings, where the bandwidth expansion mapping employs a 
scalar quantizer and transmits both the quantized value and the quantization error. Recently, a JSCC technique known as the 2:1 Shannon mapping was investigated in [11] and shown to provide very robust performance. It employs the Archimedean spiral to approximately map a point in a plane onto a point on a line. Related works on analog coding methods include [31] and [32].

To exploit the advantages of both analog and digital systems, one can allow part of the system to use digital modulation to improve robustness against severe channel conditions, while letting another part of the system use analog signaling to obtain a graceful improvement at high CSNRs. Several recent works have investigated such systems. In [19], a family of HDA systems are introduced and studied theoretically; they are shown to offer better distortion performance than purely digital systems, have a graceful performance improvement and (asymptotically) achieve the Shannon limit. An HDA system design based on vector quantization (VQ) for bandwidth expansion is investigated in [28], where an algorithm to design optimized codes and performance evaluation are presented. In [29], an HDA system for Gauss-Markov sources with bandwidth compression/expansion is given. It employs the Karhunen-Loéve transform to decorrelate the source, Turbo error correcting coding in its digital part to improve the system performance at low CSNRs, and superposition coding of the analog and digital signals. This system allows for both linear and non-linear mappings in its analog component. In [26], systematic JSCC is studied and is demonstrated to be optimal for a wide class of sources and channels. In [23], an inner distortion bound for broadcasting a single Gaussian source to two listeners over a Gaussian broadcast channel with bandwidth expansion is derived. This bound is obtained based on an HDA coding scheme, which includes one of the HDA systems of [19] and the systematic coding scheme of [26] as two special cases. In [25], systems using an HDA approach, a progressive transmission approach, and a superposition coding approach are compared for a slowly-varying fading additive white Gaussian noise (AWGN) channel. It is shown that the HDA approach has better performance than the other two methods. Most of the gain of this HDA approach is due to the presence of the linear analog part. Other HDA-based techniques are studied in [13], [20], [22], and [12] and [2]; in particular, the works of [12] and [2] study the distortion exponent (where the derived results are asymptotic in both CSNR and source dimension) for HDA coding over multipleinput multiple-output block fading channels.

In this work, we study the transmission of memoryless Gaussian sources over an AWGN channel with bandwidth compression. We investigate this problem within the HDA coding framework. We consider three HDA systems. The first system (referred to as HDA-I) is based on the recent work in [29]. For this HDA system, we first obtain an informationtheoretical (mean squared) distortion upper bound for the optimal HDA-I system with a linear analog part. As a direct consequence, we obtain a similar distortion bound for the mismatched HDA-I system where the encoder does not know the true CSNR. An optimal power allocation formula between the digital and the analog parts is obtained for this mismatched system. The second system, HDA*, and the simpler third HDA system, HDA-II, are both shown to asymptotically achieve the Shannon limit for a properly chosen power allocation between the analog and digital parts of the systems. Distortion bounds for these two systems under CSNR mismatch are also provided.

For the HDA-I system, a low-complexity and low-delay version is next designed and implemented without the use of Turbo error correcting codes (unlike the scheme of [29]) and is shown to be robust over a wide range of CSNRs. These characteristics may be particularly appealing for telemedicine and sensor networks applications where sensitive image data need to be reliably communicated from remote locations irrespective of the channel environment. The digital part of the HDA-I scheme is formed with a VQ cascaded with a binary phase-shift keying (BPSK) modulated hard-decision decoded AWGN channel. As in [28], the HDA-I system parameters (in both the digital and analog components) are optimized using an iterative algorithm similar to that for channel-optimized vector quantizer (COVQ) design. The HDA-II system is also designed and implemented without the use of channel coding; it uses a COVQ in its digital component. Simulation results indicate that while the HDA-I system provides an inferior performance to the HDA-II system for memoryless Gaussian sources, its performance is significantly better for GaussMarkov sources (decorrelated via Karhunen-Loeve processing). Comparisons are also made with purely analog and purely digital systems, as well as the system in [29].

The rest of this paper is organized as follows. In Section II, a general description of the HDA systems are given and information-theoretic bounds on the distortion are derived. Power allocation schemes for distributing the channel input power between the system's analog and digital components for both systems are also obtained. In Section III, the HDAI system design is examined in detail. Simulation results are given in Section IV. Finally, conclusions are stated in Section V.

Throughout the paper we will use the following notation. Vectors are denoted by bold-faced characters superscripted by their dimensions. Upper-case letters are used for random variables and lower-case letters for their realizations. For a given vector $\mathbf{x}^{n}=\left(x_{1}, \cdots, x_{n}\right)^{T}$, we let $\left[\mathbf{x}^{n}\right]_{1}^{k}$ and $\left[\mathbf{x}^{n}\right]_{k+1}^{n}$ denote the subvectors $\left[\mathbf{x}^{n}\right]_{1}^{k} \triangleq\left(x_{1}, \cdots, x_{k}\right)^{T}$ and $\left[\mathbf{x}^{n}\right]_{k+1}^{n} \triangleq$ $\left(x_{k+1}, \cdots, x_{n}\right)^{T}$ respectively, where $T$ denotes transposition. $\mathbb{E}(X)$ denotes the expectation of random variable $X$.

\section{INFORMATION-THEORETIC CONSIDERATIONS}

\section{A. HDA-I System}

The block diagram for the HDA-I system with bandwidth compression is depicted in Fig.1(a). Samples of a memoryless Gaussian source $\left\{X_{i}\right\}$ with zero mean and variance $\sigma_{s}^{2}>0$ are grouped into blocks of size $n$ (denoted by $\mathbf{X}^{n}$ ) and sent to a source encoder. The discrete output $I$, which is taken from a finite set of indices, is then fed to a channel encoder/modulator which produces a $k$-dimensional channel symbol $\mathbf{s}_{I}^{k}$, where $k<n$. Here $\mathbf{s}_{I}^{k}$ is taken from a finite set of possible symbols and satisfies $\mathbb{E}\left\|\mathbf{s}_{I}^{k}\right\|^{2} \leq k(1-t) P$, where $P$ is the constraint on the total input power per channel use and $t \in[0,1]$ is the power allocation coefficient for the analog part. The source 


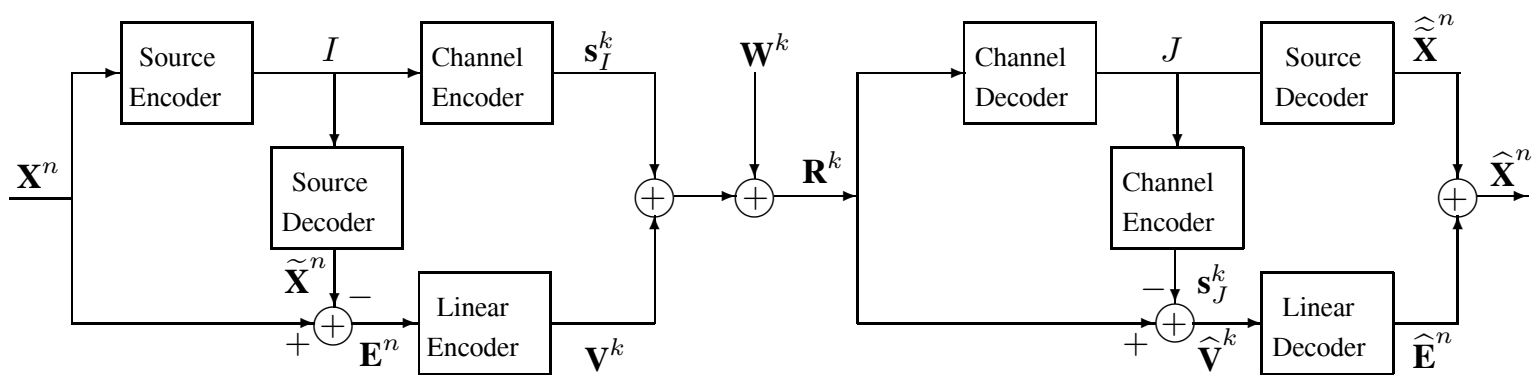

(a)

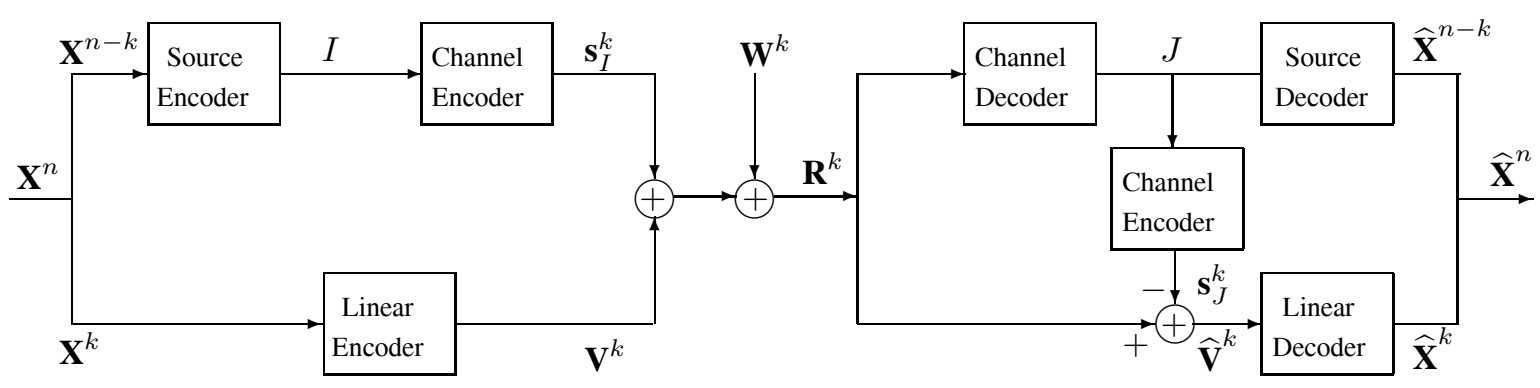

(b)

Fig. 1. HDA systems with bandwidth compression $(k<n)$ : (a) HDA-I system; (b) HDA-II system.

encoder and the channel encoder/modulator together will often be referred to as tandem source-channel encoder/modulator. The output index $I$ is also sent to a source decoder to form a reconstruction vector $\widetilde{\mathbf{X}}^{n}$, which is subtracted from $\mathbf{X}^{n}$ to form an error vector $\mathbf{E}^{n}$. The first $k$ components of $\mathbf{E}^{n}$ are further sent to a linear (analog) encoder which performs simple scaling so that the $k$-dimensional output $\mathbf{V}^{k}$ satisfies a power constraint $\mathbb{E}\left\|\mathbf{V}^{k}\right\|^{2} \leq k t P$. Now $\mathbf{s}_{I}^{k}$ and $\mathbf{V}^{k}$ are superposed and sent over a channel with AWGN $\mathbf{W}^{k}$ with per symbol noise variance $N$. The channel output $\mathbf{R}^{k}$, which is given by $\mathbf{R}^{k}=\mathbf{s}_{I}^{k}+\mathbf{V}^{k}+\mathbf{W}^{k}$, is sent to a channel decoder. The discrete output $J$ is sent to the source decoder resulting in vector $\widehat{\widetilde{\mathbf{X}}}^{n}$. Simultaneously, a channel symbol is chosen according to $J$, which is subtracted from $\mathbf{R}^{k}$. The result $\widehat{\mathbf{V}}^{k}$ is fed to the linear (analog) decoder to form an estimate $\widehat{\mathbf{E}}^{k}$. The remaining $n-k$ components of the error vector are filled with zeros to produce $\widehat{\mathbf{E}}^{n}$ which is then added to $\widehat{\widetilde{\mathbf{X}}}^{n}$ to form an estimate $\widehat{\mathbf{X}}^{n}$. The overall coding rate of this HDA-I system is $r=k / n<1$ channel uses per source sample. The system normalized mean squared error (MSE) distortion is

$$
D_{n}(N)=\frac{1}{n} \mathbb{E}\left\|\mathbf{X}^{n}-\widehat{\mathbf{X}}^{n}\right\|^{2} .
$$

For the purpose of analysis, we first consider the system's asymptotic distortion, $D(N)=\lim _{n \rightarrow \infty} D_{n}(N)$, as the block length $n$ grows without bound (assuming that the limit exists). The rate-distortion function for the memoryless Gaussian source under the squared-error distortion measure is given by $R(D)=\max \left(0,(1 / 2) \log _{2} \frac{\sigma_{s}^{2}}{D}\right)$ (bits/source sample) for any distortion value $D>0$ [27], [1]. The capacity of the AWGN channel with input power constraint $P$ and noise variance $N$ is given by $C(N)=(1 / 2) \log _{2}\left(1+\frac{P}{N}\right)$ (bits/channel use)
[27], [4]. From Shannon's lossy JSCC theorem [27], [4] for the memoryless Gaussian source-channel pair, we know that if a code has asymptotic distortion $D$, then $R(D) \leq r C(N)$ must hold. By letting $R(D)=r C(N)$, a lower bound on the asymptotic distortion of any code can be obtained. This bound is also asymptotically achievable (under the assumption that the noise variance $N$ is known by both the transmitter and the receiver), and is generally referred to as the optimal performance theoretically attainable (OPTA). It is given by

$$
D_{\text {opta }}(N) \triangleq \frac{\sigma_{s}^{2}}{\left(1+\frac{P}{N}\right)^{r}} .
$$

By examining the structure of the proposed HDA-I system in Fig.1(a), we first obtain an upper bound on $D(N)$ for optimally designed HDA-I systems.

Proposition 1: (Upper bound) For a memoryless Gaussian source with zero mean and variance $\sigma_{s}^{2}$ and an AWGN channel with noise variance $N$ (where $N$ is known at both the transmitter and the receiver), given fixed $r, P$ and $t$, there exists a sequence of HDA-I systems with asymptotic distortion $D_{h d a 1}(N)$ given by

$$
D_{h d a 1}(N)=r \frac{D_{\tan }(N)}{1+\frac{t P}{N}}+(1-r) D_{t a n}(N),
$$

where

$$
D_{\text {tan }}(N) \triangleq \frac{\sigma_{s}^{2}}{\left(1+\frac{(1-t) P}{t P+N}\right)^{r}} .
$$

Proof. The proof is given in the Appendix.

Remark: It is easy to show that $D_{h d a 1}(N)=D_{o p t a}(N)$ if and only if $t=0$. Furthermore, $D_{h d a 1}(N)=D_{\text {opta }}(N)$ for $t=r=1$. 


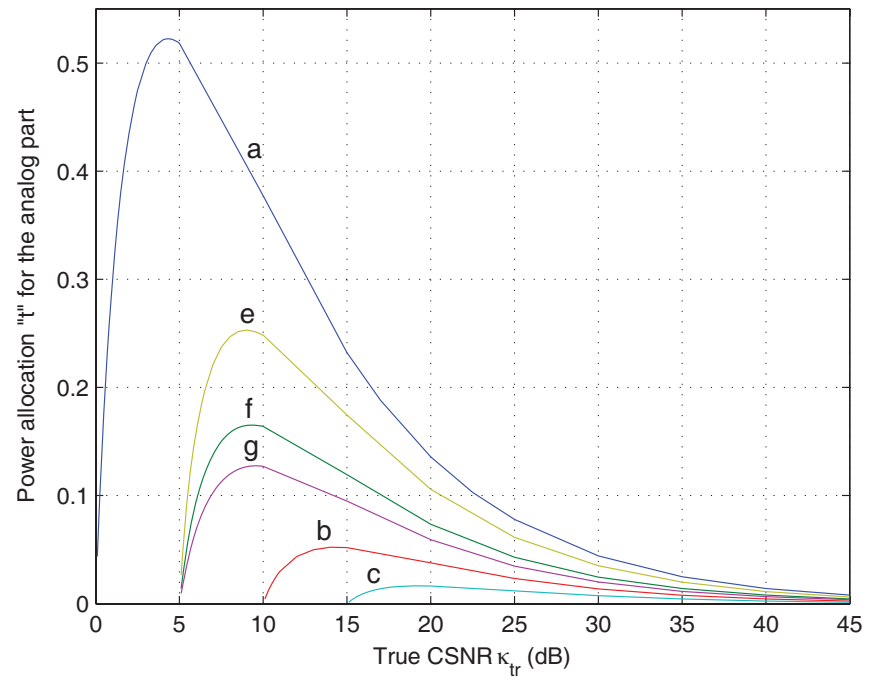

Fig. 2. The best power allocation $t$ (as a function of the true CSNR $\kappa_{t r}$ ) for different HDA-I system parameters. For curves (a), (b) and (c), $r=0.5$, $\kappa_{\text {des }}=0 \mathrm{~dB}, 10 \mathrm{~dB}$ and $15 \mathrm{~dB}$, respectively. For curves (e), (f) and (g), $\kappa_{\text {des }}=5 \mathrm{~dB}, r=0.75,0.5$ and 0.25 respectively.

We next examine the realistic situation where the AWGN variance $N$ is not known at the encoder. We assume that the encoder only knows a range of values in which the true noise variance $N_{t r}$ lies; in particular, it chooses the encoding operation for a fixed design noise variance $N_{d e s}$. The receiver, on the other hand, has full knowledge of $N_{t r}$ and adapts the decoding accordingly. For this mismatched HDA-I system, when the true noise variance $N_{t r}$ satisfies $N_{t r}<N_{d e s}$, the linear decoder can adapt to $N_{t r}$, resulting in a distortion given by $\frac{D_{t a n}\left(N_{d e s}\right)}{1+\frac{t P}{N_{t r}}}$. The asymptotic performance of the tandem coder part is still the same. We then obtain the following upper bound on the distortion:

$D_{h d a 1}^{m i s}\left(N_{t r}, N_{d e s}\right) \triangleq r \frac{D_{t a n}\left(N_{d e s}\right)}{1+\frac{t P}{N_{t r}}}+(1-r) D_{t a n}\left(N_{d e s}\right)$

where $D_{\tan }(N)$ is given in (4).

We now consider the power allocation problem for this mismatched HDA-I system with the encoder designed for $N_{d e s}$, while the true noise variance is $N_{t r}$. The best power allocation coefficient $t$ that minimizes (5) is given by the following lemma.

Proposition 2: For $N_{t r}<N_{d e s}, P$ and $r$, the power allocation coefficient $t$ which minimizes the distortion expression (5) at $N_{t r}$ is given by

$$
t=t_{1} \triangleq \frac{\sqrt{1+\frac{4\left(\kappa_{t r}-\kappa_{d e s}\right)}{(1-r) \kappa_{d e s}}}-1}{2 \kappa_{t r}}
$$

where $\kappa_{t r}=\frac{P}{N_{t r}}$ is the true CSNR and $\kappa_{\text {des }}=\frac{P}{N_{d e s}}$ is the design CSNR.

Proof. The minimizing $t$ can be easily found by setting the derivative of $D_{h d a 1}^{\text {mis }}\left(N_{t r}, N_{d e s}\right)$ with respect to $t$ to zero. The unique solution $t \in[0,1]$ is the optimal value since a direct calculation shows that the second derivative $\frac{d^{2}}{d t^{2}} D_{h d a 1}^{m i s}\left(N_{t r}, N_{d e s}\right)>0$ if $r<1$ and $N_{t r}<N_{d e s}$.

Since the optimal $t$ is a function of $N_{t r}$, it is also unavailable at the encoder. However, via a numerical study (see below) one can choose a value of $t$ which performs well for a large range of CSNRs $\kappa_{t r}$. In Fig. 2, we plot the optimal $t$ for different system parameters as a function of the true CSNR $\kappa_{t r}$. We observe the following.

- It is readily seen that as the true CSNR $\kappa_{t r}$ increases, $t$ approaches 0 . Furthermore, it is also easily seen from (6) that the rate of decay of $t$ to 0 is less than that of $1 / \kappa_{t r}$. It is easy to see that as $\kappa_{t r} \rightarrow \infty$, the distortion performance of the mismatched HDA-I system (5) approaches the constant $(1-r) D_{\text {tan }}\left(N_{\text {des }}\right)$. Curves (a), (f), (b) and (c) present the best power allocation for an HDA-I system of rate 0.5 , with design CSNR $\kappa_{\text {des }}$ of $0 \mathrm{~dB}, 5 \mathrm{~dB}, 10 \mathrm{~dB}$ and $15 \mathrm{~dB}$, respectively. They indicate that, for a system with high design CSNR (which is the case when performance at high CSNRs is the main concern), the best power allocation coefficient at various CSNR pairs $\left(\kappa_{d e s}, \kappa_{t r}\right)$ is smaller than that for the low design CSNR case, i.e., the analog part of the HDA-I system incrementally turns off as $\kappa_{d e s}$ increases without bound.

- As $\kappa_{t r}$ approaches $\kappa_{d e s}, t$ approaches 0 . Thus the optimal performance at the design CSNR is obtained by a "purely digital" design, or equivalently, by an optimal tandem coder which contains an optimal source code and an optimal channel code, as predicted by Shannon's theory [27].

- Curves (e), (f) and (g) show the best $t$ for $\kappa_{\text {des }}=5$ $\mathrm{dB}$ and coding rate of $0.75,0.5$ and 0.25 , respectively. These curves demonstrate that $t$ decreases as the coding rate $r$ decreases. Indeed, as $r$ decreases, less components of quantization error vectors are further coded via the analog part, which reduces the importance of the analog part relative to that of the tandem coding part.

In our system implementations, we fix a design CSNR $\kappa_{d e s}$ and choose an adjusted value of $t$ which is good over a large range of true CSNRs $\kappa_{t r}\left(>\kappa_{d e s}\right)$; see Section IV for details.

\section{B. HDA* and HDA-II Systems}

We note that the HDA-I system can be improved by allowing two separate source encoders in its digital component. In particular, the source vector $\mathbf{X}^{n}$ is first split into two subvectors of dimension $r n$ and $(1-r) n$, respectively, which are sent to two source encoders. The outputs of the source encoders are concatenated and fed to the (digital) channel encoder. Furthermore, the quantization error subvector resulting from encoding the first source subvector of dimension $r n$ is transmitted using the analog part (as in the HDA-I system). With this modified source encoding structure in the system, which we denote by $\mathrm{HDA}^{*}$, the quantization distortion is now determined by two source codes, unlike in the HDA-I system where a single source encoder is used. Specifically, two different per-sample quantization distortions are realized in the digital part of the $\mathrm{HDA}^{*}$ system: distortion $D_{1}$ for the first source encoder (of dimension $r n$ ), and distortion $D_{2}$ for the second source encoder (of dimension $(1-r) n$ ). It can be shown (using a proof along the same lines as that of Proposition 1), that for a given noise variance $N$ (known at both the transmitter and the receiver), fixed $P, r$, and $t \geq 0$ 
such that

$$
t \leq t^{*} \triangleq \frac{\left(1+\frac{P}{N}\right)^{r}-1}{\frac{P}{N}}
$$

we can choose $D_{1}$ and $D_{2}$ to satisfy

$$
D_{2}=\frac{D_{1}}{1+t \frac{P}{N}}
$$

and

$$
\frac{r}{2} \log _{2} \frac{\sigma_{s}^{2}}{D_{1}}+\frac{1-r}{2} \log _{2} \frac{\sigma_{s}^{2}}{D_{2}}=\frac{r}{2} \log _{2}\left(1+\frac{(1-t) P}{t P+N}\right)
$$

with $D_{1} \leq \sigma_{s}^{2}$. Then the overall asymptotic distortion (as $n$ grows to infinity), $D_{h d a^{*}}(N)$, is given by

$$
D_{h d a^{*}}(N) \triangleq r \frac{D_{1}}{1+\frac{t P}{N}}+(1-r) D_{2}=D_{2} .
$$

Substituting $D_{1}=D_{2}\left(1+t \frac{P}{N}\right)$ into (8) yields $D_{2}=\frac{\sigma_{s}^{2}}{\left(1+\frac{P}{N}\right)^{r}}$, so that

$$
D_{h d a^{*}}(N)=\frac{\sigma_{s}^{2}}{\left(1+\frac{P}{N}\right)^{r}},
$$

which is the OPTA distortion $D_{\text {opta }}(N)$. Thus for all $t \leq t^{*}$ there exists a sequence of $\mathrm{HDA}^{*}$ systems that asymptotically achieves the OPTA distortion. In particular, the choice $t=t^{*}$ results in $D_{1}=\sigma_{s}^{2}$, which means that the first $n r$ components of the source need not be quantized at all.

Furthermore, under channel mismatch (where the encoder is designed for a fixed design noise variance $N_{\text {des }}$ while the receiver knows the true noise variance $N_{t r}$, with $N_{t r}<N_{d e s}$ ), the HDA* system's distortion bound is given by

$$
D_{h d a^{*}}^{m i s}\left(N_{t r}, N_{d e s}\right) \triangleq \frac{\sigma_{s}^{2}}{\left(1+\frac{P}{N_{d e s}}\right)^{r}}(1-r+r \alpha),
$$

where

$$
\alpha \triangleq \frac{1+\frac{t P}{N_{d e s}}}{1+\frac{t P}{N_{t r}}}
$$

and

$$
0 \leq t \leq t_{2} \triangleq \frac{\left(1+\frac{P}{N_{d e s}}\right)^{r}-1}{\frac{P}{N_{d e s}}} .
$$

In Fig. 3, we compare the (asymptotic) performance of the HDA-I and HDA* systems under channel mismatch (with $r=1 / 2, \kappa_{\text {des }}=\frac{P}{N_{\text {des }}}=10 \mathrm{~dB}$ and $\kappa_{t r}=\frac{P}{N_{t r}}=20$ $\mathrm{dB}$ ) by evaluating (5) and (9) for different values of the analog power allocation $t$. The performance is in terms of the source signal-to-distortion ratio (SDR), which is defined by $\mathrm{SDR}=10 \log _{10}\left(\sigma_{s}^{2} / D\right)$ where $D$ is the MSE distortion. We remark from Fig. 3 that the $\mathrm{HDA}^{*}$ system also considerably outperforms the HDA-I system under channel mismatch. Note that the expressions of the analog power coefficients that maximize the SDR in the figure, $t_{1}$ for system HDA-I and $t_{2}$ for system HDA*, are given by (6) and (10), respectively.

Although system HDA* provides superior (asymptotic) performance over system HDA-I, it is structurally more complex as it requires the use of two source encoders in its digital component. On the one hand, this means that the encoding complexity of HDA* is less than that of HDA-I (searching in a product codebook is faster than in an unstructured codebook).

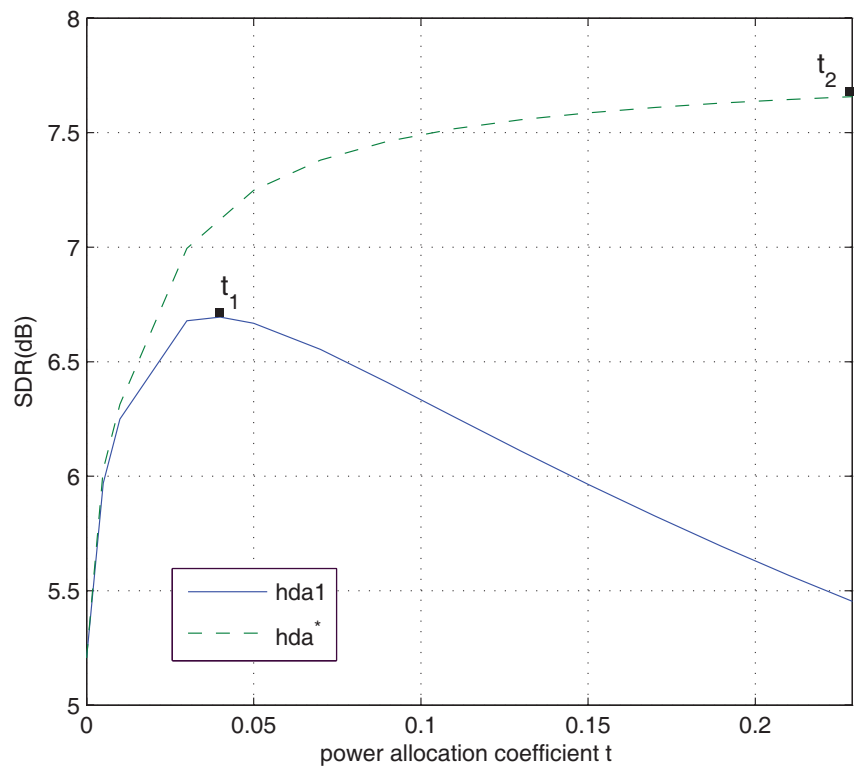

Fig. 3. SDR performance (in $\mathrm{dB}$ ) under channel mismatch for the HDAI system (curve hda1) and the improved HDA* (curve hda*) system with $r=\frac{1}{2}$, design CSNR $\kappa_{\text {des }}=10 \mathrm{~dB}$ and true CSNR $\kappa_{t r}=20 \mathrm{~dB} ; t_{1}$ and $t_{2}$ are the optimal analog power coefficients for systems HDA-I and HDA*, respectively.

On the other hand, the joint optimization of the system components is already quite complex in the conceptually simpler HDA-I system (see Section III), and the presence of two codebooks makes this joint optimization procedure even more difficult for HDA*. For this latter reason, we will only implement a simplified version of the $\mathrm{HDA}^{*}$ system, called HDA-II, which is depicted in Fig. 1(b).

In this system, instead of quantizing the first $r n$ source symbols and sending the quantization error via the analog part, the first $r n$ symbols of $\mathbf{X}^{n}$ are directly transmitted using analog coding (without the quantization part). The last $(1-r) n$ source symbols are (as in the HDA* system) quantized and sent over the digital component of the system (see Fig. 1.(b)). Analogously to systems HDA-I and HDA*, one can show that the following distortion, $D_{h d a 2}(N)$, can be asymptotically achieved by the HDA-II system:

$$
D_{h d a 2}(N) \triangleq r \frac{\sigma_{s}^{2}}{1+\frac{t P}{N}}+(1-r) \frac{\sigma_{s}^{2}}{\left(1+\frac{\frac{(1-t) P}{N}}{1+\frac{t P}{N}}\right)^{\frac{r}{1-r}}} .
$$

Remark: Note that system HDA-II can still (asymptotically) achieve the OPTA distortion: by setting $t=t^{*}$, we obtain that $D_{\text {hda } 2}(N)=D_{\text {opta }}(N)$.

Furthermore, the channel mismatch distortion bound for the HDA-II system, is given by

$D_{h d a^{*}}^{m i s}\left(N_{t r}, N_{d e s}\right) \triangleq r \frac{\sigma_{s}^{2}}{1+\frac{t P}{N_{t r}}}+(1-r) \frac{\sigma_{s}^{2}}{\left(1+\frac{\frac{(1-t) P}{N_{d e s}}}{1+\frac{t P}{N_{d e s}}}\right)^{\frac{r}{1-r}}}$

where $N_{t r}<N_{\text {des }}$. 


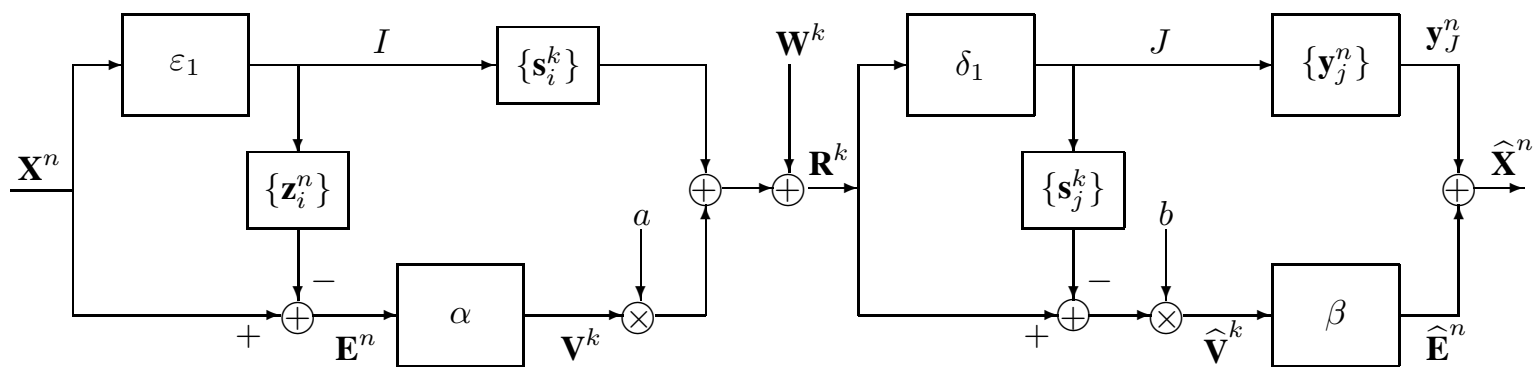

Fig. 4. Proposed HDA-I system design with bandwidth compression.

\section{HDA-I SYSTEM DESIGN}

We next consider a concrete implementation of the HDA-I scheme in Fig. 1(a). This system, which has low-complexity and low-delay as it avoids the use of channel coding in its digital part, is depicted in Fig. 4, and it employs VQ cascaded with BPSK modulation in the digital part, and uses linear coding in the analog part.

\section{A. System Description}

The upper part, referred to as the digital part, is formed by a VQ cascaded with a binary symmetric channel (BSC) without the use of channel coding. An output index $I$ of the $k$-bit $n$-dimensional VQ encoder $\varepsilon_{1}$ is assigned a $k$-dimensional channel symbol $\mathbf{s}_{I}^{k}$ from a set $\left\{\mathbf{s}_{i}^{k}\right\}$ of $2^{k}$ possible symbols. The index $I$ also chooses a vector $\mathbf{z}_{I}^{n}$ from the encoder codebook $\left\{\mathbf{z}_{i}^{n}\right\}$, which is subtracted from $\mathbf{X}^{n}$ to form the error vector $\mathbf{E}^{n}$.

In the ideal case, for a memoryless source, the optimal source code (in the sense of asymptotically achieving the ratedistortion curve) splits source vectors into two asymptotically orthogonal components, the quantizer output and the quantization error (see, e.g., [19]). Furthermore, for memoryless Gaussian sources, the distribution of the quantization error is also approximately Gaussian as $n \rightarrow \infty$ (see Appendix). In the HDA-I system with linear analog coding, since the output of the linear analog encoder is just a scaled version of the quantization error, we model (as discussed in the Appendix) the output of the linear encoder by a vector of independent Gaussian random variable with variance $t P$ which is independent of the source. Hence, for the digital part, a BSC is realized by using hard decision decoding on the BPSK-modulated AWGN channel with input power $(1-t) P$ and noise variance $t P+N_{\text {des }}$. Consequently, if the BPSK signals take values in $\{+\sqrt{(1-t) P},-\sqrt{(1-t) P}\}$, the transition probabilities $\left\{P_{J \mid I}(j \mid i)\right\}$ of the BSC are $P_{J \mid I}(j \mid i)=$ $q^{d_{H}(i, j)}(1-q)^{k-d_{H}(i, j)}$, where $d_{H}(i, j)$ denotes the Hamming distance between the binary representations of the integers $i$ and $j$, and $q=Q\left(\sqrt{\kappa_{d i g}}\right)$ is the crossover probability, where $\kappa_{d i g} \triangleq \frac{(1-t) \kappa_{\text {des }}}{t \kappa_{\text {des }}+1}$ is the effective CSNR of the digital part and $Q(x) \stackrel{1}{=} \frac{1}{\sqrt{2 \pi}} \int_{x}^{\infty} e^{-t^{2} / 2} d t$. We remark that any memoryless modulation constellation can be used besides BPSK modulation. We choose BPSK modulation because it is simple and it performs comparatively well at low CSNRs.

Given an input error vector $\mathbf{E}^{n}$, the mapping $\alpha$ simply takes the first $k$ components of $\mathbf{E}^{n}$ and forms a scaled vector $\mathbf{V}^{k}$ (to satisfy the average power constraint), which is added to $\mathbf{s}_{I}^{k}$ and sent over the AWGN channel. The received vector $\mathbf{R}^{k}$ is first fed to decoder $\delta_{1}$ (which is a simple binary hard-decision demodulator), resulting in index $J$, and the corresponding reproduction $\mathbf{y}_{J}^{n}$ is chosen through a lookup table. The channel symbol $\mathbf{s}_{J}^{k}$ is then subtracted from $\mathbf{R}^{k}$ and scaled by a constant $b$, forming an estimate $\widehat{\mathbf{V}}^{k}$. The mapping $\beta$ expands the message $\mathbf{V}^{k}$ back to $n$ dimensions, by padding it with zeros in the corresponding locations. The resulting $\widehat{\mathbf{E}}^{n}$ is added back to $\mathbf{y}_{J}^{n}$ to form the reproduction $\widehat{\mathbf{X}}^{n}$.

\section{B. System Design}

For a total input power $P$, a fixed power allocation $t$ and a design noise variance $N_{\text {des }}$, we derive an iterative training algorithm to optimize the source digital transmitter (both source encoder and source decoder) and both the digital decoder codebook and the analog decoder. Given an arbitrary encoder $\varepsilon_{1},\left\{\mathbf{z}_{i}^{n}\right\},\left\{\mathbf{s}_{i}^{n}\right\},\left\{\mathbf{y}_{j}^{n}\right\}$, and $a$ and $b$, the end-to-end average distortion can be expressed as (13) (see next page). To considerably simplify the derivation of our results, we make the following assumptions. We assume that $a$ is chosen such that the power constraint

$$
a^{2} \mathbb{E}\left\|\left[\mathbf{X}^{n}\right]_{1}^{k}-\left[\mathbf{z}_{I}^{n}\right]_{1}^{k}\right\|^{2}=k t P
$$

is satisfied, that $\mathbf{W}^{k}$ is uncorrelated with both $\left[\mathbf{y}_{J}^{n}\right]_{1}^{k}$ and $\left[\mathbf{s}_{J}^{n}\right]_{1}^{k}$ and that $E\left[\mathbf{W}^{k} \mid J\right]=0$. It is worthwhile to point out that the last two assumptions are valid when the channel is noiseless; hence they provide good approximations in the high CSNR regime and their merit will be assessed when evaluating the performance of the resulting HDA-I system design in Section IV.

Then distortion $D_{n}^{1}\left(N_{\text {des }}\right)$ can be expressed as shown on the next page, where the second equality follows from our assumption that $\mathbf{W}^{k}$ is uncorrelated from $\left[\mathbf{y}_{J}^{n}\right]_{1}^{k}$ and $\left[\mathbf{s}_{J}^{n}\right]_{1}^{k}$.

Lemma 1: Fix a set of encoder regions $\left\{Q_{i}\right\}$ of $\epsilon_{1}$. For any digital decoder codebook $\left\{\mathbf{y}_{j}^{n}\right\}$ and $b$, the digital source decoder codebook $\left\{\left[\mathbf{z}_{i}^{n}\right]_{1}^{k}\right\}$ that minimizes the average distortion (13) is given by

$$
\left[\mathbf{z}_{i}^{n}\right]_{1}^{k}=\left[\overline{\mathbf{y}}_{i}^{n}\right]_{1}^{k}+b\left(\mathbf{s}_{i}^{k}-\overline{\mathbf{s}}_{i}^{k}\right), \quad i=0, \cdots, 2^{k}-1 .
$$

For any $\left\{\left[\mathbf{z}_{i}^{n}\right]_{1}^{k}\right\}$, the average distortion (13) is minimized by choosing $b$ and $\left\{\mathbf{y}_{j}^{n}\right\}$ as follows:

$$
b=\frac{\mathbb{E}\left[\left(\left[\mathbf{X}^{n}\right]_{1}^{k}-\mathbb{E}\left[\left[\mathbf{X}^{n}\right]_{1}^{k} \mid J\right]\right)^{T} \mathbf{U}^{k}\right]}{k N_{\text {des }}+\mathbb{E}\left\|\mathbf{U}^{k}\right\|^{2}},
$$




$$
\begin{aligned}
D_{n}\left(N_{\text {des }}\right)=\frac{1}{n} \mathbb{E}\left\|\mathbf{X}^{n}-\widehat{\mathbf{X}}^{n}\right\|^{2} \\
=\frac{1}{n} \mathbb{E} \|\left(\begin{array}{c}
{\left[\mathbf{X}^{n}\right]_{1}^{k}} \\
{\left[\mathbf{X}^{n}\right]_{k+1}^{n}}
\end{array}\right)-\left(\begin{array}{c}
{\left[\mathbf{y}_{J}^{n}\right]_{1}^{k}} \\
{\left[\mathbf{y}_{J}^{n}\right]_{k+1}^{n}}
\end{array}\right) \\
\quad-\left(\begin{array}{c}
b\left(a\left(\left[\mathbf{X}^{n}\right]_{1}^{k}-\left[\mathbf{z}_{I}^{n}\right]_{1}^{k}\right)+\mathbf{s}_{I}^{k}+\mathbf{W}^{k}-\mathbf{s}_{J}^{k}\right) \\
0
\end{array}\right) \|^{2} \\
=\frac{1}{n} \underbrace{\mathbb{E}\left\|\left[\mathbf{X}^{n}\right]_{1}^{k}-\left[\mathbf{y}_{J}^{n}\right]_{1}^{k}-b\left(a\left(\left[\mathbf{X}^{n}\right]_{1}^{k}-\left[\mathbf{z}_{I}^{n}\right]_{1}^{k}\right)+\mathbf{s}_{I}^{k}+\mathbf{W}^{k}-\mathbf{s}_{J}^{k}\right)\right\|^{2}}_{\triangleq D_{n}^{1}\left(N_{d e s}\right)} \\
+\frac{1}{n} \underbrace{\mathbb{E}\left\|\left[\mathbf{X}^{n}\right]_{k+1}^{n}-\left[\mathbf{y}_{J}^{n}\right]_{k+1}^{n}\right\|^{2}}_{\triangleq D_{n}^{2}\left(N_{\text {des }}\right)}
\end{aligned}
$$

$$
\begin{aligned}
D_{n}^{1}\left(N_{\text {des }}\right) & \\
= & \frac{1}{n} \mathbb{E}\left\|\left[\mathbf{X}^{n}\right]_{1}^{k}-\left[\mathbf{y}_{J}^{n}\right]_{1}^{k}-b\left(a\left(\left[\mathbf{X}^{n}\right]_{1}^{k}-\left[\mathbf{z}_{I}^{n}\right]_{1}^{k}\right)+\mathbf{s}_{I}^{k}+\mathbf{W}^{k}-\mathbf{s}_{J}^{k}\right)\right\|^{2} \\
= & \frac{1}{n} \mathbb{E}\left\|\left[\mathbf{X}^{n}\right]_{1}^{k}-\left[\mathbf{y}_{J}^{n}\right]_{1}^{k}-b\left(\mathbf{s}_{I}^{k}-\mathbf{s}_{J}^{k}\right)-b a\left(\left[\mathbf{X}^{n}\right]_{1}^{k}-\left[\mathbf{z}_{I}^{n}\right]_{1}^{k}\right)\right\|^{2} \\
& +\frac{1}{n} b^{2} \mathbb{E}\left\|\mathbf{W}^{k}\right\|^{2} \\
= & \frac{1}{n} \mathbb{E}\left\|\left[\mathbf{X}^{n}\right]_{1}^{k}-\left[\mathbf{y}_{J}^{n}\right]_{1}^{k}-b\left(\mathbf{s}_{I}^{k}-\mathbf{s}_{J}^{k}\right)\right\|^{2} \\
& +\frac{1}{n} b^{2} a^{2} \mathbb{E}\left\|\left[\mathbf{X}^{n}\right]_{1}^{k}-\left[\mathbf{z}_{I}^{n}\right]_{1}^{k}\right\|^{2} \\
& -2 a b \frac{1}{n} \mathbb{E}\left[\left(\left[\mathbf{X}^{n}\right]_{1}^{k}-\left[\mathbf{y}_{J}^{n}\right]_{1}^{k}-b\left(\mathbf{s}_{I}^{k}-\mathbf{s}_{J}^{k}\right)\right)^{T}\left(\left[\mathbf{X}^{n}\right]_{1}^{k}-\left[\mathbf{z}_{I}^{n}\right]_{1}^{k}\right)\right] \\
& +\frac{k}{n} b^{2} N_{\text {des }} \\
= & \frac{1}{n} \mathbb{E}\left\|\left[\mathbf{X}^{n}\right]_{1}^{k}-\left[\mathbf{y}_{J}^{n}\right]_{1}^{k}-b\left(\mathbf{s}_{I}^{k}-\mathbf{s}_{J}^{k}\right)\right\|^{2}+\frac{k}{n} b^{2} t P \\
& -2 a b \frac{1}{n} \mathbb{E}\left[\left(\left[\mathbf{X}^{n}\right]_{1}^{k}-\left[\mathbf{y}_{J}^{n}\right]_{1}^{k}-b\left(\mathbf{s}_{I}^{k}-\mathbf{s}_{J}^{k}\right)\right)^{T}\left(\left[\mathbf{X}^{n}\right]_{1}^{k}-\left[\mathbf{z}_{I}^{n}\right]_{1}^{k}\right)\right] \\
& +\frac{k}{n} b^{2} N_{\text {des }}
\end{aligned}
$$

$$
\begin{aligned}
{\left[\mathbf{y}_{j}^{n}\right]_{1}^{k} } & =\sum_{i=0}^{2^{k}-1} P_{I \mid J}(i \mid j)\left(\left[\overline{\mathbf{x}}_{i}^{n}\right]_{1}^{k}-b a\left(\left[\overline{\mathbf{x}}_{i}^{n}\right]_{1}^{k}-\left[\mathbf{z}_{i}^{n}\right]_{1}^{k}\right)\right) \\
-b( & \left.\sum_{i=0}^{2^{k}-1} P_{I \mid J}(i \mid j) \mathbf{s}_{i}^{k}-\mathbf{s}_{j}^{k}\right), j=0, \cdots, 2^{k}-1, \\
{\left[\mathbf{y}_{j}^{n}\right]_{k+1}^{n}=} & \sum_{i=0}^{2^{k}-1} P_{I \mid J}(i \mid j)\left[\overline{\mathbf{x}}_{i}^{n}\right]_{k+1}^{n}, \\
& j=0, \cdots, 2^{k}-1,
\end{aligned}
$$

where, see (19) on the next page, and $p\left(\mathbf{x}^{n}\right)$ is the pdf of $\mathbf{x}^{n}$.

Proof. We first focus on how the digital source decoder codebooks $\left\{\left[\mathbf{z}_{i}^{n}\right]_{1}^{k}\right\}$ should be chosen to minimize the distortion $D_{n}\left(N_{\text {des }}\right)$ (note that the $\left\{\left[\mathbf{z}_{i}^{n}\right]_{k+1}^{n}\right\}$ are not needed since we only transmit the first $k$ error components). We note that the only term in the above expression of $D_{n}^{1}\left(N_{\text {des }}\right)$ that can be influenced by changing $\left\{\left[\mathbf{z}_{i}^{n}\right]_{1}^{k}\right\}$ is the third one. We have (20)-(23) (see next page), where (21) holds from the fact that
$\left[\mathbf{X}^{n}\right]_{1}^{k} \rightarrow i \rightarrow\left[\mathbf{y}_{j}^{n}\right]_{1}^{k}, \mathbf{s}_{j}^{k}$ forms a Markov chain, and (23) holds by the Cauchy-Schwarz inequality. For arbitrary given $\left\{\mathbf{y}_{j}^{n}\right\}$ and $b$, equality holds when we choose $\left\{\left[\mathbf{z}_{i}^{n}\right]_{1}^{k}\right\}$ as in (15), thus minimizing the distortion $D_{n}^{1}\left(N_{\text {des }}\right)$. Next, consider how the digital decoder codebook $\left\{\mathbf{y}_{j}^{n}\right\}$ should be chosen to minimize the average distortion $D_{n}\left(N_{\text {des }}\right)$ in (13). Recall that

$$
\begin{aligned}
D_{n}^{1}\left(N_{\text {des }}\right)= & \frac{1}{n} \mathbb{E} \|\left(\left[\mathbf{X}^{n}\right]_{1}^{k}-b a\left(\left[\mathbf{X}^{n}\right]_{1}^{k}-\left[\mathbf{z}_{I}^{n}\right]_{1}^{k}\right)\right. \\
& \left.\quad-b\left(\mathbf{s}_{I}^{k}-\mathbf{s}_{J}^{k}+\mathbf{W}^{k}\right)\right)-\left[\mathbf{y}_{J}^{n}\right]_{1}^{k} \|^{2}, \\
D_{n}^{2}\left(N_{\text {des }}\right)= & \frac{1}{n} \mathbb{E}\left\|\left[\mathbf{X}^{n}\right]_{k+1}^{n}-\left[\mathbf{y}_{J}^{n}\right]_{k+1}^{n}\right\|^{2} .
\end{aligned}
$$

Thus, for arbitrary $\left\{\left[\mathbf{z}_{i}^{n}\right]_{1}^{k}\right\}$ and $b$, the $\left\{\mathbf{y}_{j}^{n}\right\}$ which minimize the average distortion (13) are obtained by letting $\left\{\mathbf{y}_{j}^{n}\right\}$ represent the minimum mean square error (MMSE) estimator (see (24) on the next page), where (24) follows from our assumption that $E\left[\mathbf{W}^{k} \mid J\right]=0$. Choosing $\left\{\mathbf{y}_{j}^{n}\right\}$ as above, and defining $\mathbf{U}^{k}$ as in (19), the distortion can be rewritten as shown after (24) on the next page. Minimizing the above 


$$
\begin{aligned}
& \mathbf{U}^{k} \triangleq a\left(\left[\mathbf{X}^{n}\right]_{1}^{k}-\mathbb{E}\left[\left[\mathbf{X}^{n}\right]_{1}^{k} \mid J\right]-\left[\mathbf{z}_{I}^{n}\right]_{1}^{k}+\mathbb{E}\left[\left[\mathbf{z}_{I}^{n}\right]_{1}^{k} \mid J\right]\right) \\
&+\mathbf{s}_{I}^{k}-\mathbf{s}_{J}^{k}-\mathbb{E}\left[\mathbf{s}_{I}^{k}-\mathbf{s}_{J}^{k} \mid J\right] \\
& \overline{\mathbf{x}}_{i}^{n} \triangleq \mathbb{E}\left[\mathbf{X}^{n} \mid I=i\right]=\int_{\mathbf{x}^{n} \in Q_{i}} \mathbf{x}^{n} p\left(\mathbf{x}^{n}\right) d \mathbf{x}^{n} \\
& \overline{\mathbf{y}}_{i}^{n} \triangleq \mathbb{E}\left[\mathbf{y}_{J}^{n} \mid I=i\right]=\sum_{j=1}^{2^{k}-1} P_{J \mid I}(j \mid i) \mathbf{y}_{j}^{n} \\
& \overline{\mathbf{s}}_{i}^{k} \triangleq \mathbb{E}\left[\mathbf{s}_{J}^{k} \mid I=i\right]=\sum_{j=1}^{2^{k}-1} P_{J \mid I}(j \mid i) \mathbf{s}_{j}^{k} \\
& P_{I \mid J}(i \mid j) \triangleq \operatorname{Pr}(I=i \mid J=j)=P_{J \mid I}(j \mid i) P_{I}(i) / P_{J}(j) \\
& P_{I}(i) \triangleq \operatorname{Pr}(I=i)=\operatorname{Pr}\left(\mathbf{X}^{n} \in Q_{i}\right) \\
& P_{J}(j) \triangleq \operatorname{Pr}(J=j)=\sum_{i=1}^{2^{k}-1} P_{I}(i) P_{J \mid I}(j \mid i)
\end{aligned}
$$

$$
\begin{aligned}
\mathbb{E}[ & \left.\left(\left[\mathbf{X}^{n}\right]_{1}^{k}-\left[\mathbf{y}_{J}^{n}\right]_{1}^{k}-b\left(\mathbf{s}_{I}^{k}-\mathbf{s}_{J}^{k}\right)\right)^{T}\left(\left[\mathbf{X}^{n}\right]_{1}^{k}-\left[\mathbf{z}_{I}^{n}\right]_{1}^{k}\right)\right] \\
= & \mathbb{E}\left(\left[\mathbf{X}^{n}\right]_{1}^{k^{T}}\left[\mathbf{X}^{n}\right]_{1}^{k}\right)-\mathbb{E}\left(\left[\mathbf{X}^{n}\right]_{1}^{k^{T}}\left[\mathbf{z}_{I}^{n}\right]_{1}^{k}\right) \\
& -\mathbb{E}\left[\left(\left[\mathbf{y}_{J}^{n}\right]_{1}^{k}+b\left(\mathbf{s}_{I}^{k}-\mathbf{s}_{J}^{k}\right)\right)^{T}\left(\left[\mathbf{X}^{n}\right]_{1}^{k}-\left[\mathbf{z}_{I}^{n}\right]_{1}^{k}\right)\right] \\
= & \mathbb{E}\left\|\left[\mathbf{X}^{n}\right]_{1}^{k}\right\|^{2}-\sum_{i=0}^{2^{k}-1} P_{I}(i) \\
& \cdot\left(\left(\left[\overline{\mathbf{y}}_{i}^{n}\right]_{1}^{k}+b\left(\mathbf{s}_{i}^{k}-\overline{\mathbf{s}}_{i}^{k}\right)\right)^{T}\left(\left[\overline{\mathbf{x}}_{i}^{n}\right]_{1}^{k}-\left[\mathbf{z}_{i}^{n}\right]_{1}^{k}\right)-\left[\overline{\mathbf{x}}_{i}^{n}\right]_{1}^{k^{T}}\left[\mathbf{z}_{i}^{n}\right]_{1}^{k}\right) \\
= & \mathbb{E}\left[\left(\left[\mathbf{X}^{n}\right]_{1}^{k}-\left[\overline{\mathbf{y}}_{I}^{n}\right]_{1}^{k}-b\left(\mathbf{s}_{I}^{k}-\overline{\mathbf{s}}_{I}^{k}\right)\right)^{T}\left(\left[\mathbf{X}^{n}\right]_{1}^{k}-\left[\mathbf{z}_{I}^{n}\right]_{1}^{k}\right)\right] \\
\leq & \sqrt{\mathbb{E}\left\|\left[\mathbf{X}^{n}\right]_{1}^{k}-\left[\overline{\mathbf{y}}_{I}^{n}\right]_{1}^{k}-b\left(\mathbf{s}_{I}^{k}-\overline{\mathbf{s}}_{I}^{k}\right)\right\|^{2} \mathbb{E}\left\|\left[\mathbf{X}^{n}\right]_{1}^{k}-\left[\mathbf{z}_{I}^{n}\right]_{1}^{k}\right\|^{2}}
\end{aligned}
$$

$$
\begin{aligned}
{\left[\mathbf{y}_{j}^{n}\right]_{1}^{k}=} & \mathbb{E}\left[\left[\mathbf{X}^{n}\right]_{1}^{k}-b a\left(\left[\mathbf{X}^{n}\right]_{1}^{k}-\left[\mathbf{z}_{I}^{n}\right]_{1}^{k}\right)-b\left(\mathbf{s}_{I}^{k}-\mathbf{s}_{J}^{k}+\mathbf{W}^{k}\right) \mid J=j\right]=\sum_{i=0}^{2^{k}-1} P_{I \mid J}(i \mid j)\left(\left[\overline{\mathbf{x}}_{i}^{n}\right]_{1}^{k}-b a\left(\left[\overline{\mathbf{x}}_{i}^{n}\right]_{1}^{k}-\left[\mathbf{z}_{i}^{n}\right]_{1}^{k}\right)\right) \\
& -b\left(\sum_{i=0}^{2^{k}-1} P_{I \mid J}(i \mid j) \mathbf{s}_{i}^{k}-\mathbf{s}_{j}^{k}\right),\left[\mathbf{y}_{j}^{n}\right]_{k+1}^{n}=\mathbb{E}\left[\left[\mathbf{X}^{n}\right]_{k+1}^{n} \mid J=j\right]=\sum_{i=0}^{2^{k}-1} P_{I \mid J}(i \mid j)\left[\overline{\mathbf{x}}_{i}^{n}\right]_{k+1}^{n}
\end{aligned}
$$

$$
\begin{aligned}
D_{n}\left(N_{\text {des }}\right)= & \frac{1}{n} \mathbb{E}\left\|\mathbf{X}^{n}-\mathbb{E}\left[\mathbf{X}^{n} \mid J\right]\right\|^{2} \\
& -\frac{1}{n} 2 b \mathbb{E}\left[\left(\left[\mathbf{X}^{n}\right]_{1}^{k}-\frac{1}{n} \mathbb{E}\left[\left[\mathbf{X}^{n}\right]_{1}^{k} \mid J\right]\right)^{T} \mathbf{U}^{k}\right] \\
& +\frac{1}{n} b^{2} \mathbb{E}\left\|\mathbf{U}^{k}\right\|^{2}+\frac{k}{n} b^{2} N_{\text {des }} .
\end{aligned}
$$


distortion by solving $\frac{\partial D_{n}\left(N_{\text {des }}\right)}{\partial b}=0$ yields the expression of $b$ given by (16).

Lemma 2: For a fixed digital decoder codebook $\left\{\mathbf{y}_{j}^{n}\right\}, a$ and $b$, fixed $\left\{\left[\mathbf{z}_{i}^{n}\right]_{1}^{k}\right\}$ as in (15), the optimal encoder regions $\left\{Q_{i}\right\}$ for $\epsilon_{1}$ are given as follows:

$$
\begin{aligned}
Q_{i}= & \left\{\mathbf{x}^{n} \in \mathbb{R}^{n}: i=\arg \min _{l}\left((a b-1)^{2}\left\|\left[\mathbf{x}^{n}\right]_{1}^{k}-\left[\mathbf{z}_{l}^{n}\right]_{1}^{k}\right\|^{2}\right.\right. \\
& \left.\left.+h_{l}+\sum_{j=0}^{2^{k}-1}\left\|\left[\mathbf{x}^{n}\right]_{k+1}^{n}-\left[\mathbf{y}_{j}^{n}\right]_{k+1}^{n}\right\|^{2} P_{J \mid I}(j \mid l)\right)\right\}(25)
\end{aligned}
$$

where

$$
h_{l} \triangleq \mathbb{E}\left[\left\|\left[\mathbf{y}_{J}^{n}\right]_{1}^{k}+b\left(\mathbf{s}_{I}^{k}-\mathbf{s}_{J}^{k}\right)\right\|^{2} \mid I=l\right]-\mathbb{E}\left[\left\|\left[\mathbf{z}_{I}^{n}\right]_{1}^{k}\right\|^{2}\right] \text {. }
$$

Proof. The distortion $D_{n}^{1}\left(N_{\text {des }}\right)$ in (13) can be written as shown on the next page, where $h_{i}$ is defined as (26). Combining $D_{n}^{1}\left(N_{d e s}\right)$ above with $D_{n}^{2}\left(N_{d e s}\right)$ in (13) yields

$$
\begin{aligned}
& D_{n}\left(N_{\text {des }}\right) \\
& =\sum_{i=0}^{2^{k}-1} \int_{Q_{i}} d\left[\mathbf{x}^{n}\right]_{1}^{k} p\left(\left[\mathbf{x}^{n}\right]_{1}^{k}\right)\left\{(a b-1)^{2}\left\|\left[\mathbf{x}^{n}\right]_{1}^{k}-\left[\mathbf{z}_{i}^{n}\right]_{1}^{k}\right\|^{2}\right. \\
& \left.\quad+h_{i}+\sum_{j=0}^{2^{k}-1}\left\|\left[\mathbf{x}^{n}\right]_{k+1}^{n}-\left[\mathbf{y}_{j}^{n}\right]_{k+1}^{n}\right\|^{2} P_{J \mid I}(j \mid i)\right\} \\
& \quad+k b^{2} N_{\text {des }} .
\end{aligned}
$$

Therefore, the optimal encoder regions are given by (25).

\section{Some Special Cases}

In Proposition 2, we derived the optimal power allocation coefficient $t$ (with respect to $D_{h d a}^{m i s}\left(N_{t r}, N_{d e s}\right)$ ) as a function of the design CSNR $\kappa_{\text {des }}$. Here we discuss the special cases of high and low $\kappa_{\text {des }}$ regimes and examine how the power allocation coefficient $t$ and the system distortion change with $\kappa_{\text {des }}$ from the design point of view.

Assuming that the system is designed for a CSNR of $\kappa_{\text {des }}=P / N_{\text {des }}$ and a power allocation coefficient $t$, the digital channel has an effective CSNR of $\kappa_{d i g}=\frac{(1-t) \kappa_{d e s}}{t \kappa_{d e}+1}$, which means that the BSC transition probabilities $P_{J \mid I}(j \mid i)$ are calculated with the latter CSNR. Assume also that $\left\{Q_{i}\right\}$, $\left\{\left[\mathbf{z}_{i}^{n}\right]_{1}^{k}\right\},\left\{\mathbf{y}_{j}^{n}\right\}$, and $b$ are chosen according to the results of Section III-B. We consider the following situations.

- Low noise case, $\kappa_{\text {des }} \rightarrow \infty$. In this case, $\kappa_{\text {dig }} \approx \frac{1-t}{t}$ and the $P_{J \mid I}(j \mid i)$ 's no longer depend on $\kappa_{\text {des }}$. Since decoding the analog signal is dependent on the correct decoding of the digital signal, we can allocate more transmission power to the digital part (decrease $t$ ) to increase $\kappa_{\text {dig }}$, as long as $t P \gg N_{\text {des }}$. As a result, the distortion due to the digital transmission part decreases, which in turn makes the analog part more useful. This choice of $t$ is consistent with the result of Proposition 2 (see Fig. 2). As more power is allocated to the digital part (e.g., as $t$ decreases), $P_{J \mid I}(j \mid i) \rightarrow 0$ for $j \neq i$, hence, $\overline{\mathbf{s}}_{I}^{k} \rightarrow \mathbf{s}_{I}^{k}$, $\left[\mathbf{z}_{I}^{n}\right]_{1}^{k} \rightarrow\left[\overline{\mathbf{y}}_{I}^{n}\right]_{1}^{k} \rightarrow\left[\mathbf{y}_{I}^{n}\right]_{1}^{k}$, and $b \rightarrow \frac{1}{a}$. As a result, the encoder region $\left\{Q_{i}\right\}$ in (25) is simplified to $Q_{I}=\left\{\mathbf{x}^{n} \in\right.$ $\left.\mathbb{R}^{n}: I=\arg \min _{l}\left(\left\|\left[\mathbf{x}^{n}\right]_{k+1}^{n}-\left[\mathbf{y}_{l}^{n}\right]_{k+1}^{n}\right\|^{2}\right)\right\}$ since $(a b-$
$1)^{2} \rightarrow 0$ and $h_{l} \rightarrow 0$. Thus the dominant distortion is the non-recoverable quantization error from the rest of the $n-k$ components of the source vectors. This observation is also justified by Proposition 1, where the first term of (3) goes to zero as $t P / N \rightarrow \infty$ (note that as $\kappa_{d e s} \rightarrow \infty$, we also have $\kappa_{t r} \rightarrow \infty$ since we assume that $\kappa_{t r}>\kappa_{\text {des }}$ ).

- High noise case, $\kappa_{\text {des }} \rightarrow 0$. In this case $b \rightarrow 0$, which means that we will not decode the analog signal because of its bad quality. Moreover, $\left[\mathbf{z}_{I}^{n}\right]_{1}^{k} \rightarrow\left[\overline{\mathbf{y}}_{I}^{n}\right]_{1}^{k}$ in (15) and $\mathbf{y}_{j}^{n} \rightarrow \sum_{i} P_{I \mid J}(i \mid j) \overline{\mathbf{x}}_{i}^{n}$ in (17), (18). Since

$$
\begin{aligned}
\sum_{i} P_{I \mid J}(i \mid j) \overline{\mathbf{x}}_{i}^{n} & =\sum_{i} P_{I \mid J}(i \mid j) \mathbb{E}\left[\mathbf{X}^{n} \mid I=i\right] \\
& =\sum_{i} P_{I \mid J}(i \mid j) \mathbb{E}\left[\mathbf{X}^{n} \mid I=i, J=j\right] \\
& =\mathbb{E}\left[\mathbf{X}^{n} \mid J=j\right],
\end{aligned}
$$

we have $\mathbf{y}_{J}^{n} \rightarrow \mathbb{E}\left[\mathbf{X}^{n} \mid J\right]$, which means that the digital part approaches a COVQ [9]. In this case, it is best to allocate all the power to the digital part.

\section{Training Algorithm}

The results of Lemmas 1 and 2 can be used to formulate an iterative training algorithm as in [28], [33] for codebooks design. The algorithm is summarized as follows: (1) Given the design noise variance $N_{d e s}$, total power $P$, power allocation coefficient $t$, and two thresholds $\gamma_{1}, \gamma_{2}$, calculate the corresponding transition probabilities $P_{J \mid I}(j \mid i)$ of the digital channel. Initialize the encoder regions ${ }^{1}\left\{Q_{i}\right\}$; (2) Determine the encoder centroids $\left\{\overline{\mathbf{x}}_{i}^{n}\right\}$ and the probabilities $\left\{P_{I}(i)\right\}$, initialize $\left[\mathbf{z}_{I}^{n}\right]_{1}^{k}=\left[\overline{\mathbf{x}}_{I}^{n}\right]_{1}^{k}$, initialize $a$ to satisfy the power constraint; (3) Iteratively compute $b,\left\{\mathbf{y}_{j}^{n}\right\}$ and $\left\{\left[\mathbf{z}_{i}^{n}\right]_{1}^{k}\right\}$ using Lemma 1, update $a$ after each iteration to satisfy power constraint, and stop when the changes of the codebooks $\left\{\mathbf{y}_{j}^{n}\right\}$ and $\left\{\left[\mathbf{z}_{i}^{n}\right]_{1}^{k}\right\}$ fall below the threshold $\gamma_{1}$; (4) Redefine the encoder regions $\left\{Q_{i}\right\}$ using Lemma 2, update $a$ again, and estimate the average distortion; (5) Repeat steps (3) and (4) until the change of the average distortion falls below the threshold $\gamma_{2}$. In the simulations, $\gamma_{1}=10^{-5}$ and $\gamma_{2}=10^{-8}$ were used. We have the following remarks.

- Optimizing $\left\{\left[\mathbf{z}_{i}^{n}\right]_{1}^{k}\right\},\left\{\mathbf{y}_{j}^{n}\right\}$ and $b$ jointly is very complex. Instead, in the design we use Lemma 1 for an iterative approach similar to the one in [28]. First, we initialize $\left[\mathbf{z}_{I}^{n}\right]_{1}^{k}=\left[\overline{\mathbf{x}}_{I}^{n}\right]_{1}^{k}$. Then, we compute $b$ using (16), and compute $\left\{\mathbf{y}_{j}^{n}\right\}$ using (17) and (18). We next update $\left\{\left[\mathbf{z}_{i}^{n}\right]_{1}^{k}\right\}$ using (15) with the new value of $b$ and $\left\{\mathbf{y}_{j}^{n}\right\}$. The iterative algorithm is stopped when the changes of the codebooks $\left\{\left[\mathbf{z}_{i}^{n}\right]_{1}^{k}\right\}$ and $\left\{\mathbf{y}_{j}^{n}\right\}$ fall below a certain threshold.

- In our derivation, we assume that the power constraint (14) is satisfied with equality at all times. Strictly speaking, there is no guarantee for this to hold at all iterations. Therefore, convergence is not guaranteed. In our design, the coefficient $a$ is updated after each computation of $\left\{\left[\mathbf{z}_{i}^{n}\right]_{1}^{k}\right\}$ to satisfy the power constraint. Our experimental

\footnotetext{
${ }^{1}$ Here we use the Voronoi regions of a VQ trained for a noiseless channel for the same source under consideration. An alternative way is to use the encoder of a COVQ [9] trained for the same digital channel $\left.\left\{P_{J \mid I}(j \mid i)\right\}\right)$.
} 


$$
\begin{aligned}
D_{n}^{1}\left(N_{\text {des }}\right) & \\
= & \frac{1}{n} \mathbb{E}\left\|\left[\mathbf{X}^{n}\right]_{1}^{k}-\left[\mathbf{z}_{I}^{n}\right]_{1}^{k}\right\|^{2}+\frac{1}{n} \mathbb{E}\left\|\left[\mathbf{y}_{J}^{n}\right]_{1}^{k}+b\left(\mathbf{s}_{I}^{k}-\mathbf{s}_{J}^{k}\right)\right\|^{2} \\
& -\frac{1}{n} \mathbb{E}\left\|\left[\mathbf{z}_{I}^{n}\right]_{1}^{k}\right\|^{2}+\frac{k}{n} b^{2} N_{\text {des }} \\
& -\frac{1}{n} 2 a b \mathbb{E}\left[\left(\left[\mathbf{X}^{n}\right]_{1}^{k}-\left[\overline{\mathbf{y}}_{I}^{n}\right]_{1}^{k}-b\left(\mathbf{s}_{I}^{k}-\overline{\mathbf{s}}_{I}^{k}\right)\right)^{T}\left(\left[\mathbf{X}^{n}\right]_{1}^{k}-\left[\mathbf{z}_{I}^{n}\right]_{1}^{k}\right)\right] \\
& +\frac{1}{n} a^{2} b^{2} \mathbb{E}\left\|\left[\mathbf{X}^{n}\right]_{1}^{k}-\left[\mathbf{z}_{I}^{n}\right]_{1}^{k}\right\|^{2} \\
= & \frac{1}{n}(a b-1)^{2} \mathbb{E}\left\|\left[\mathbf{X}^{n}\right]_{1}^{k}-\left[\mathbf{z}_{I}^{n}\right]_{1}^{k}\right\|^{2} \\
& +\frac{1}{n} \mathbb{E}\left\|\left[\mathbf{y}_{J}^{n}\right]_{1}^{k}+b\left(\mathbf{s}_{I}^{k}-\mathbf{s}_{J}^{k}\right)\right\|^{2}-\frac{1}{n} \mathbb{E}\left\|\left[\mathbf{z}_{I}^{n}\right]_{1}^{k}\right\|^{2}+\frac{k}{n} b^{2} N_{\text {des }} \\
= & \sum^{k}-1 \\
& \sum_{i=0} \frac{1}{n} \int_{Q_{i}}\left\{(a b-1)^{2}\left\|\left[\mathbf{x}^{n}\right]_{1}^{k}-\left[\mathbf{z}_{i}^{n}\right]_{1}^{k}\right\|^{2}+h_{i}\right\} \\
& \cdot p\left(\left[\mathbf{x}^{n}\right]_{1}^{k}\right) d\left[\mathbf{x}^{n}\right]_{1}^{k}+\frac{k}{n} b^{2} N_{\text {des }}
\end{aligned}
$$

studies suggest that the iterative algorithm does converge to a stable solution.

- In our design, all the codebooks are precomputed off line. During encoding, the digital encoder finds $\left\{Q_{i}\right\}$ using Lemma 2. It is easily seen from (25) that, $\left\{h_{l}\right\}$ can be precomputed. Given the input vector $\mathbf{x}^{n}$, most of the computation needed to find the encoder region involves the full COVQ-type search over the codebook $\left\{\mathbf{y}_{j}^{n}\right\}$ restricted to the last $n-k$ dimensions, i.e., we need to compute $\sum_{j=0}^{2^{k}-1}\left\|\left[\mathbf{x}^{n}\right]_{k+1}^{n}-\left[\mathbf{y}_{j}^{n}\right]_{k+1}^{n}\right\|^{2} P_{J \mid I}(j \mid l)$. Thus, we can see that when a moderate block size $n$ is used (e.g., $n=24$ is used in the simulation of Section IV), the digital encoding part has low computational complexity and low delay. For the decoding part, since we use hard-decision demodulation, and the digital decoder codebook $\left\{\mathbf{y}_{j}^{n}\right\}$ is precomputed off line, we only need to perform table-lookup decoding. Thus, the digital decoding complexity is low. As for the analog part, only $k$ multiplications are needed for linear encoding/decoding.

\section{Simulation Results}

We evaluate the SDR performance for the transmission of both i.i.d. Gaussian sources and Gauss-Markov sources over the AWGN channel via the HDA-I and HDA-II systems, designed without the use of channel coding in their digital component.

For the i.i.d. Gaussian source, the source samples are grouped into vectors of dimension $n=24$, and transmitted at an overall rate of $1 / 2$ channel use per source sample. We implement the HDA-I design system using the training algorithm described in the previous section. Specifically, for a fixed input power $P=1$ and design noise variance $N_{\text {des }}=0.1$ (thus $\kappa_{\text {des }}=P / N_{\text {des }}=10$ ), the training algorithm is implemented to generate the source digital transmitter and both the digital decoder codebook and the analog decoder. In light of Proposition 2 and curve (b) of Fig. 2, we choose $t=0.05$ (this choice of $t$ is expected to give good performance

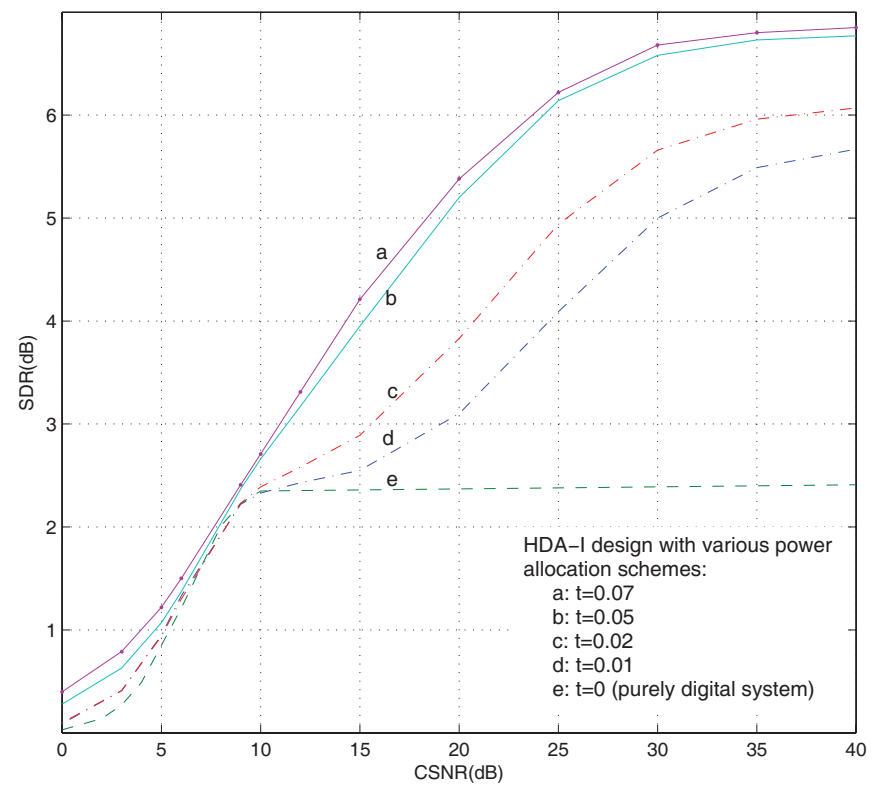

Fig. 5. SDR performance (in $\mathrm{dB}$ ) of optimized HDA-I systems for various power allocation coefficients $t$; i.i.d. Gaussian source over the AWGN channel, $\kappa_{\text {des }}=10 \mathrm{~dB}, r=1 / 2$ channel use/source sample.

in the true CSNR range of 12 to $20 \mathrm{~dB}$ for the asymptotically achievable system). Apart from this choice of $t$, we carried out simulations with other choices of $t \in[0,1]$ for the purpose of comparison. Motivated by a broadcast scenario, we assume (e.g., as in [28]) that the encoder is optimized for a given power allocation and fixed design CSNR $\kappa_{\text {des }}$, i.e., $\varepsilon_{1}$ and $\left\{\mathbf{z}_{i}^{n}\right\}$ are designed for a fixed $\kappa_{\text {des }}$, while the decoder knows the true CSNR $\kappa_{t r}$ and adapts to it, i.e., $\left\{\mathbf{y}_{j}^{n}\right\}$ and $b$ are adapted to $\kappa_{t r}$. We also implement the HDA-II system using a simple power scaling encoder and MMSE decoding adapted to the true CSNR $\kappa_{t r}$ in its analog component, and a rate-1 COVQ source encoder (designed for $\kappa_{\text {des }}=P / N_{\text {des }}=10$ ) and a COVQ decoder adapted to $\kappa_{t r}$ in its digital component. 


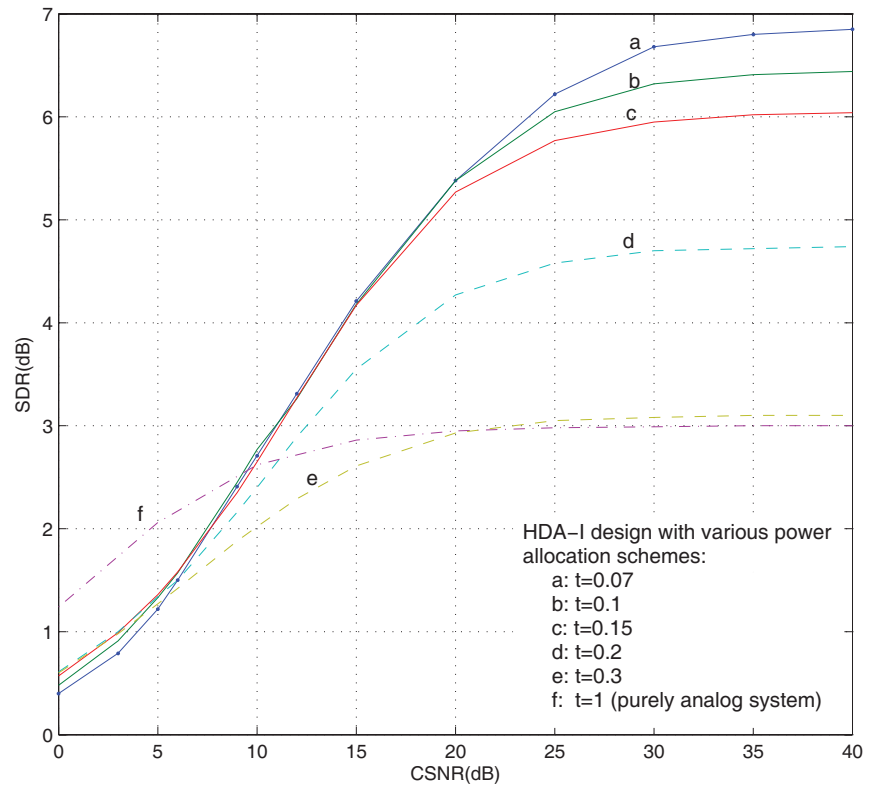

Fig. 6. SDR performance (in $\mathrm{dB}$ ) of optimized HDA-I systems for various power allocation coefficients $t$; i.i.d. Gaussian source over the AWGN channel, $\kappa_{\text {des }}=10 \mathrm{~dB}, r=1 / 2$ channel use/source sample.

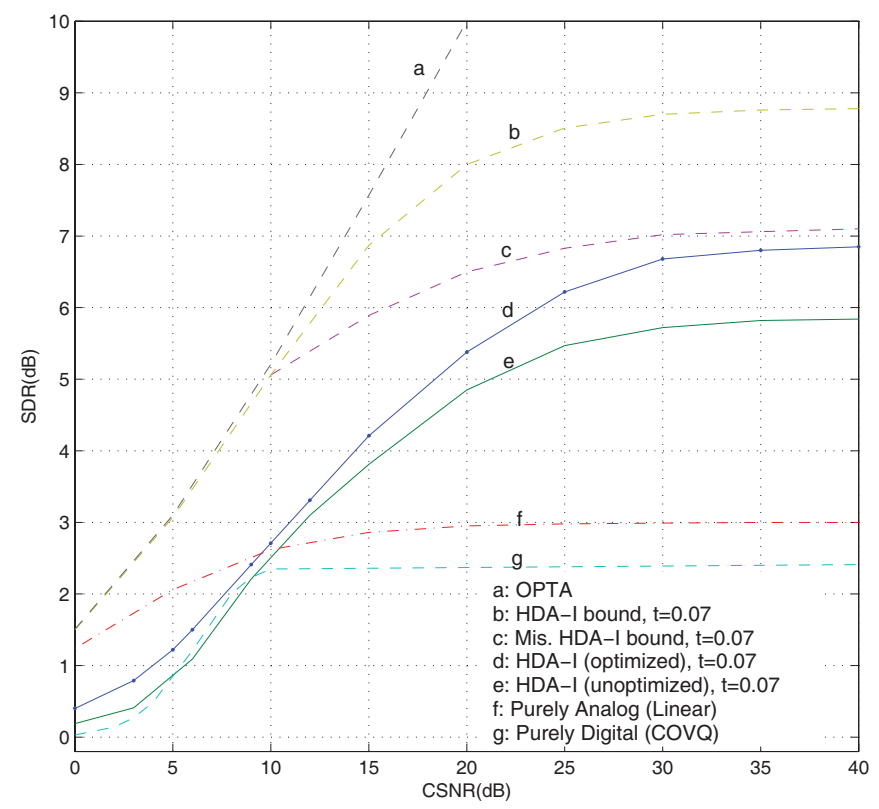

Fig. 7. SDR performance (in $\mathrm{dB}$ ) of the optimized HDA-I, the unoptimized HDA-I, the purely digital and the purely analog systems; i.i.d. Gaussian source over the AWGN channel, $r=1 / 2$ channel use/source sample. For the HDA-I and purely digital systems, $\kappa_{\text {des }}=10 \mathrm{~dB}$.

For the Gauss-Markov source, we first employ KarhunenLoéve processing to the source vector. The HDA-I system then transmits the first 12 error symbols with the largest variance via the analog part. For the HDA-II system, the first 12 source coefficients with largest variance are coded using the digital method, the remaining 12 coefficients are transmitted using the analog coding; since this is slightly different from the original structure of HDA-II introduced in Section II, we refer to it as HDA-II* in the simulation results presented in Fig. 10 (note that HDA-II* performs better than HDA-II for the case of a

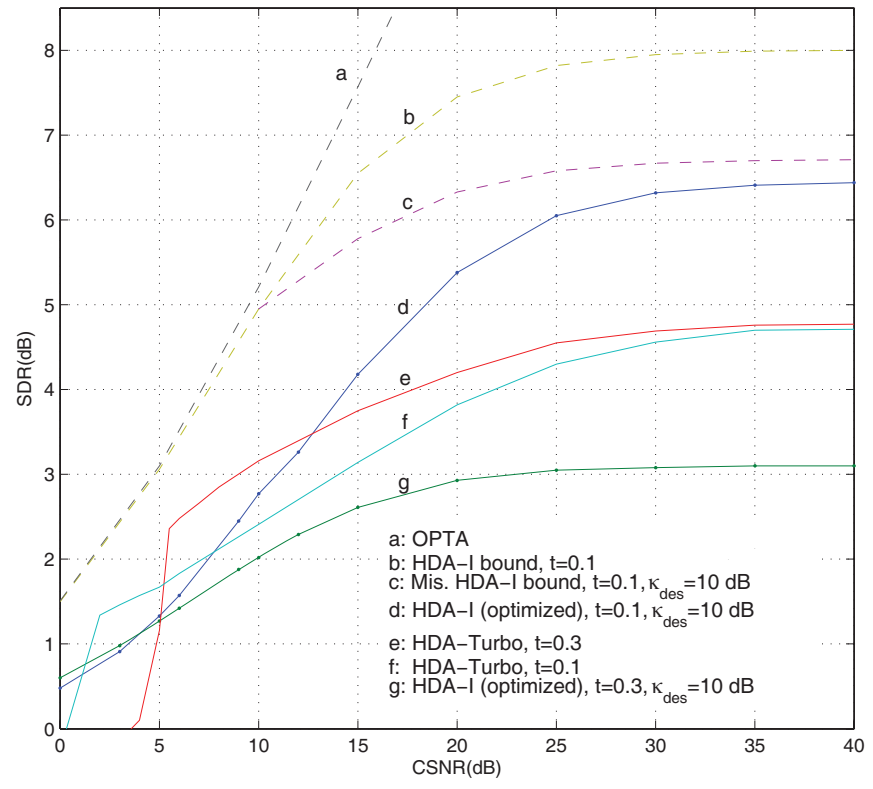

Fig. 8. SDR performance (in $\mathrm{dB}$ ) of various HDA systems; i.i.d. Gaussian source over the AWGN channel, $r=1 / 2$ channel use/source sample.

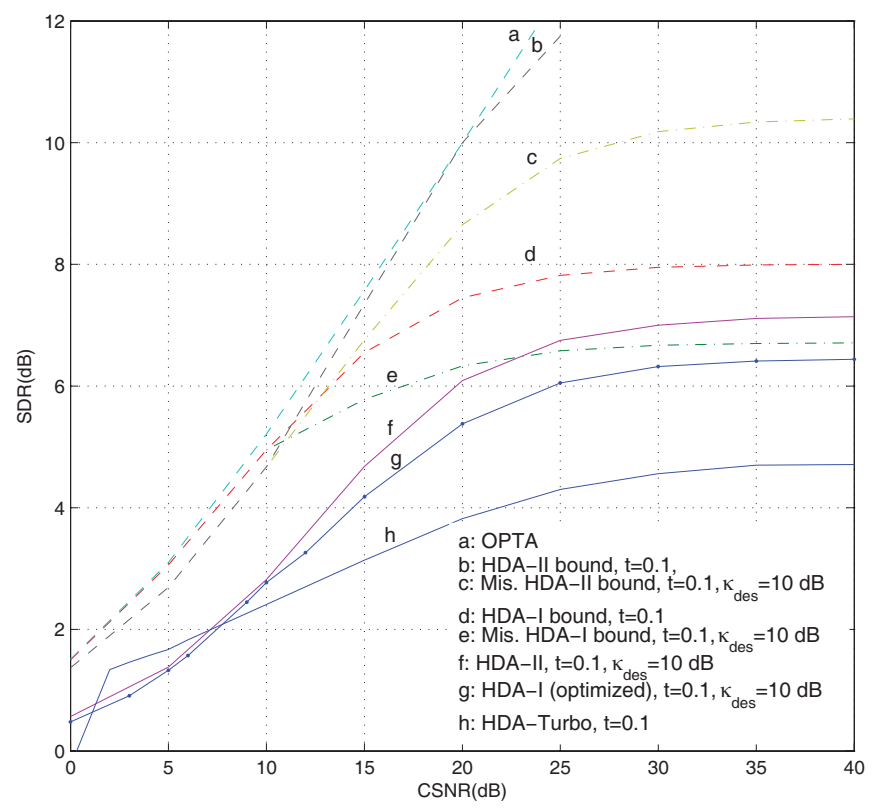

Fig. 9. SDR performance (in $\mathrm{dB}$ ) of various HDA systems; i.i.d. Gaussian source over the AWGN channel, $r=1 / 2$ channel use/source sample.

\section{Gauss-Markov source).}

We present simulation results for the optimized HDA-I system with various power allocation coefficients $t$ and the HDA-II system, as well as an unoptimized HDA-I system, a purely digital system, a purely analog system and the HDATurbo system of [29]. All systems have a transmission rate of $1 / 2$ channel use per source sample.

- The optimized HDA-I system performance is shown in Figs. 5-10 for $\kappa_{\text {des }}=10 \mathrm{~dB}$ and various values of $t$.

- The HDA-II system performance is shown in Figs. 9 and 10 for $\kappa_{\text {des }}=10 \mathrm{~dB}$ and $t=0.1$.

- The unoptimized HDA-I system uses the Linde-Buzo- 


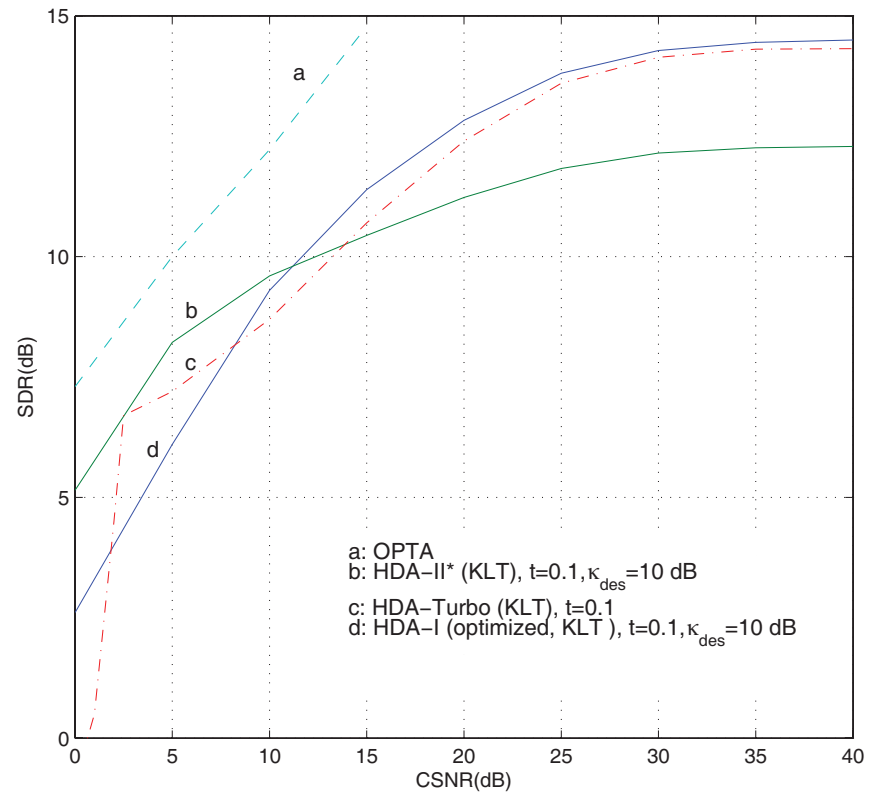

Fig. 10. SDR performance (in $\mathrm{dB}$ ) of various HDA systems; GaussianMarkov source (with correlation coefficient 0.9) over the AWGN channel, $r=1 / 2$ channel use/source sample.

Gray (LBG) algorithm [18] to design the digital encoder $\varepsilon_{1}$ and $\left\{\mathbf{z}_{i}^{n}\right\}$, and applies a linear encoder to the analog part. The digital decoder codebook $\left\{\mathbf{y}_{j}^{n}\right\}$ is adapted to the true CSNR $\kappa_{t r}$, and a linear MMSE decoder (also assuming knowledge of $\kappa_{t r}$ ) is applied to the analog part; its performance is shown in Fig. 7 for $t=0.07$.

- The purely digital system, which solely employs the digital part of the HDA-I system, uses a COVQ source encoder [9] and a COVQ decoder codebook $\left\{\mathbf{y}_{j}^{n}\right\}$ adapted to the true CSNR $\kappa_{t r}$; its performance is shown in Fig. 7 for $\kappa_{d e s}=10 \mathrm{~dB}$.

- The purely analog system, which solely employs the analog part of the HDA-I system, transmits only half of each source vector using linear coding and employs a linear MMSE decoder with knowledge of the true CSNR; its performance is shown in Fig. 7.

- For the HDA-Turbo system of [29], the digital part consists of a 24-dimensional 6-bit VQ designed using the LBG algorithm, and a high-delay $(k=768, n=1536)$ rate $1 / 2$ Turbo encoder with generator $(37,21)$ (punctured to rate $1 / 2$ ) and a random interleaver, and the analog part employs the same methods as the proposed HDAI schemes. The digital decoder $\left\{\mathbf{y}_{j}\right\}$ and the analog decoder also has knowledge of $\kappa_{t r}$; its performance is shown in Fig. 8 for $t=0.1$ and $t=0.3$ and in Fig. 10 for $t=0.1$.

All systems are trained with 300,000 vectors, and tested with a different set of 100,000 vectors. For comparison purposes, we also present the following theoretical curves: the OPTA curve (2) for the memoryless Gaussian source (shown in Figs. 7-9) and the OPTA curve for the Gauss-Markov source (shown in Fig. 10); the HDA-I bounds for both matched and mismatched cases (shown in Figs. 7-9), described for a given $t$ by (3) and (5), respectively; and the HDA-II bounds for both matched and mismatched cases (shown in Fig. 9), described for a given $t$ by (11) and (12), respectively. We can observe the following:

- Figs. 5-6 indicate that the power allocation plays an important role in the performance of the optimized HDAI system, especially for CSNRs above the design CSNR of $10 \mathrm{~dB}$. Although we choose $t=0.05$ based on Proposition 2, $t=0.07$ turns out to be the best power allocation shown by the simulation results. In particular, the SDR increases as $t$ increases from $t=0$ (which is equivalent to the purely digital system) to about $t=0.07$ (see Fig. 5) and then declines as $t$ varies from $t=0.07$ to $t=1$ (which is equivalent to the purely analog system). While the optimal power allocation provided by Proposition 2 is derived for the ideal case (which assumes infinite block size), and the above numerical results are derived using a block size of 24 , we note that the best choice (around $t=0.07$ ) obtained by the numerical study is consistent with the value $t=0.05$ suggested by Fig. 2 . Another interesting observation is that when the true CSNR falls below $10 \mathrm{~dB}\left(\kappa_{\text {des }}\right)$, the SDR performance gets better as $t$ increases. This is because the digital part degrades drastically when $\kappa_{t r}<\kappa_{\text {des }}$ (usually, the better the digital part performs at the design CSNR, the more drastic is its performance degradation for lower CSNRs).

- We observe from Fig. 7 that for $t=0.07$, the optimized HDA-I system outperforms the unoptimized HDA-I system at all CSNRs. Moreover, it obtains a gain of $1 \mathrm{~dB}$ over the unoptimized HDA-I system, and is within 0.3 $\mathrm{dB}$ of the performance bound for the mismatched HDA-I system at high CSNRs (e.g., for CSNR $\geq 30 \mathrm{~dB}$ ). The HDA-I systems present a smooth and robust performance for most CSNRs, and provide substantial improvements over the purely digital system from medium to high CSNRs. They also outperform the purely analog system for a wide range of CSNRs. We also note that the performance saturates at around $35 \mathrm{~dB}$.

- In Fig. 8, we compare the optimized HDA-I system with the HDA-Turbo system of [29] for $t=0.1$ and $t=0.3$. We remark that for a proper choice of $t$, e.g., for $t=0.1$, the optimized HDA-I system outperforms the HDA-Turbo system for CSNR $\geq 13 \mathrm{~dB}$, and obtain a large gain for medium to high CSNRs. This behavior can be explained as follows. During the linear encoding process, we discard half of the components of each quantization error vector. For memoryless sources, all components of the error vectors have approximately the same variance. Since the optimized HDA-I system has higher quantization rate than that of the HDA-Turbo system (the HDA-I scheme does not employ channel coding while the HDA-Turbo system uses a rate $1 / 2$ Turbo code), each component of the quantization error vector has a smaller variance than the corresponding quantization error component in the HDA-Turbo system. As a result, the distortion introduced in the optimized HDA-I system by this dropping-off process in the analog part is less severe than that for the HDA-Turbo system. On the other hand, the Turbo code plays an important 
role for CSNRs ranging from 5 to $10 \mathrm{~dB}$. For CSNRs over $10 \mathrm{~dB}$, channel coding becomes superfluous and most of the system distortion is due to quantization noise. Fig. 8 shows that in the CSNR range of 25 to $40 \mathrm{~dB}$, the optimized HDA-I system has a gain around $1.5 \mathrm{~dB}$ over the HDA-Turbo system.

- In Figs. 9 and 10, we compare the HDA-I and the HDA-II/HDA-II* systems with $t=0.1$ for both i.i.d. Gaussian and Gauss-Markov sources. As predicted by the theoretical curves of Fig. 3, the HDA-II bounds are superior to the HDA-I bounds for the memoryless Gaussian source (even though both simulated systems do not use channel coding). Note from the figure, that for $\mathrm{CSNR}=19 \mathrm{~dB}$, curve (b) of the HDA-II bound (11) meet the OPTA performance (curve (a)). This is indeed expected from our study in Section II, since at for this CSNR, we obtain from (7) that $t^{*}=0.1$, and hence we should have that $D_{h d a 2}(N)=D_{\text {opta }}(N)$. Furthermore, in the simulation results, the HDA-II system (curve (f) in Fig. 9), outperforms the HDA-I system (curve (g)) for a wide range of CSNR, with about a $0.6 \mathrm{~dB}$ SDR gain for CSNR varying from 20 to $40 \mathrm{~dB}$.

However, in the simulation result for the Gauss-Markov source, the HDA-II* system is inferior to the HDA-I system for CSNRs above $11 \mathrm{~dB}$; compare curves (d) and (b) in Fig. 10. The SDR gain of HDA-I over HDA-II* is substantial for high CSNR's (about $2.5 \mathrm{~dB}$ ). Furthermore, the HDA-II* system performs worse than the HDA-Turbo system for CSNRs above $14 \mathrm{~dB}$. We conclude that for the memoryless Gaussian source, the HDA-II performs better than the HDA-I system, while for the Gauss-Markov source, the HDA-I system provides a superior performance. Thus if the source is known to be Gaussian, but it is not known whether it is memoryless or Gauss-Markovian, the HDA-I system would serve as a good compromise for a low-complexity and robust HDA system.

\section{CONCLUSION}

Three HDA joint source-channel systems with bandwidth compression for the reliable communication of Gaussian sources over AWGN channels are studied. All systems have a simple linear analog coding component. Information-theoretic distortion upper bounds (under both matched and mismatched channel conditions) for the case of memoryless Gaussian sources are established. It is shown that two of the HDA systems ( $\mathrm{HDA}^{*}$ and HDA-II) can asymptotically achieve OPTA under matched channel conditions for optimally chosen power allocations between the analog and digital parts of the systems, thus theoretically outperforming the first scheme, HDA-I. Then, a practical HDA-I scheme which employs a VQ cascaded with BPSK modulation in the digital part is designed and implemented. A training algorithm is presented to iteratively optimize the source digital transmitter (both source encoder and source decoder) and both the digital decoder codebook and the analog decoder. A system design of the HDA-II scheme is also conducted. Both implemented HDA-I and HDA-II schemes are similar to the system considered in
[29], but they are simpler as they do not use Turbo errorcorrecting coding. Numerical results show that both HDA schemes offer a robust and graceful performance improvement for a wide range of CSNRs (medium to high CSNRs), and substantially outperforms purely digital and purely analog systems for a large range of CSNRs. The HDA-II is superior to the HDA-I system for the memoryless Gaussian source; however, for the Gauss-Markov source, the HDA-I system performs substantially better than the HDA-II system. The advantages of the HDA schemes are as follows: (1) they have low complexity and low delay; (2) they guarantee a graceful performance improvement for high CSNRs; (3) for the HDA-I system, the joint source-channel design of the codebooks enables smooth degradation for medium CSNRs. In [33, Chapter 3], an image communication application that illustrates the effectiveness of HDA coding is presented by combining the HDA-I system with the bandwidth expansion system of Skoglund et al. [28].

\section{APPENDIX: ProOF OF PROPOSITION 1}

First we give an informal derivation of the upper bound, and then we provide the outline of a rigorous derivation which uses common randomization at the encoder and the decoder. Some straightforward but tedious details will be omitted. For the source encoder and decoder in the upper "digital" part of the system let $\left(\varphi_{e}^{(n)}, \varphi_{d}^{(n)}\right)$ be a sequence of source codes (vector quantizers) with encoder $\varphi_{e}^{(n)}: \mathbb{R}^{n} \rightarrow$ $\left\{1, \ldots, 2^{n R}\right\}$ and decoder $\varphi_{d}^{(n)}:\left\{1, \ldots, 2^{n R}\right\} \rightarrow \mathbb{R}^{n}$, having rate $R=\frac{r}{2} \log \left(1+\frac{(1-t) P}{t P+N}\right)$ bits per source sample. We choose $\left(\varphi_{e}^{(n)}, \varphi_{d}^{(n)}\right)$ so that it asymptotically achieves the distortionrate function at rate $R$ of the i.i.d. Gaussian source with zero mean and variance $\sigma_{s}^{2}$. Thus letting $\widetilde{\mathbf{X}}^{n}=\varphi_{d}^{(n)}\left(\varphi_{e}^{(n)}\left(\mathbf{X}^{n}\right)\right)$ and $D_{n} \triangleq \frac{1}{n} \mathbb{E}\left\|\mathbf{X}^{n}-\widetilde{\mathbf{X}}^{n}\right\|^{2}$, we have

$$
\lim _{n \rightarrow \infty} D_{n}=\sigma_{s}^{2} 2^{-2 R}=\frac{\sigma_{s}^{2}}{\left(1+\frac{(1-t) P}{t P+N}\right)^{r}}=D_{t a n}(N) .
$$

The output index $I=\varphi_{e}^{(n)}\left(\mathbf{X}^{n}\right)$ from the source encoder is fed to the channel encoder which operates on blocks of $k=r n$ channel symbols. The sequence of channel codes $\left(\psi_{e}^{(k)}, \psi_{d}^{(k)}\right)$ with encoder $\psi_{e}^{(k)}:\left\{1, \ldots, 2^{n R}\right\} \rightarrow \mathbb{R}^{k}$ and decoder $\psi_{d}^{(k)}:$ $\mathbb{R}^{k} \rightarrow\left\{1, \ldots, 2^{n R}\right\}$ has rate

$$
\frac{n}{k} R=\frac{R}{r}=\frac{1}{2} \log \left(1+\frac{(1-t) P}{t P+N}\right)
$$

bits per channel use. This is the capacity of an AWGN channel with noise variance $t P+N$ and input power constraint $(1-t) P$, and we choose the channel code to satisfy this power constraint and such that its error probability is asymptotically (i.e., as $k \rightarrow \infty$ ) zero when it is used on this AWGN channel. Letting $\mathbf{E}^{n} \triangleq \mathbf{X}^{n}-\widetilde{\mathbf{X}}^{n}$, the linear encoder-decoder pair $\left(\alpha^{(n)}, \beta^{(n)}\right)$ is defined as

$$
\begin{aligned}
& \mathbf{V}^{k} \triangleq \alpha^{(n)}\left(\mathbf{E}^{n}\right)=\sqrt{\frac{t P}{D_{n}}}\left[\mathbf{E}^{n}\right]_{1}^{k}, \\
& \widehat{\mathbf{E}}^{n} \triangleq \beta^{(n)}\left(\widehat{\mathbf{V}}^{k}\right)=\left(\frac{\sqrt{t P D_{n}}}{t P+N}\left(\widehat{\mathbf{V}}^{k}\right)^{T},\left(\mathbf{0}^{n-k}\right)^{T}\right)^{T}
\end{aligned}
$$


where $\left[\mathbf{E}^{n}\right]_{1}^{k}$ denotes the first $k$ components of $\mathbf{E}^{n}$. Since the source code asymptotically achieves the rate-distortion function, one can easily show using a standard information theoretic argument that the normalized relative entropy (Kullback Leibler divergence) [4] between $\mathbf{E}^{n}$ and an $n$-dimensional Gaussian random vector with i.i.d. components of zero mean and variance $D_{\tan }(N)$ converges to zero as $n \rightarrow \infty$. This indicates that the distribution of $\mathbf{E}^{n}$ is well approximated by that of the Gaussian vector for large $n$. It is also easy to show that $\mathbf{E}^{n}$ and $\widetilde{\mathbf{X}}^{n}$ are (asymptotically) uncorrelated (see, e.g., [19, Lemma 1]). To simplify the informal derivation, let us assume that the following stronger versions of these approximations hold: (i) $\mathbf{E}^{n}$ is independent of $\widetilde{\mathbf{X}}^{n}$; (ii) $\mathbf{E}^{n}$ is Gaussian with independent components of zero mean and equal variance $D_{n}$.

Note that since $I$ is a function of $\widetilde{\mathbf{X}}^{n}$, these assumptions imply that the channel codeword $\mathbf{s}_{I}^{k}=\psi_{e}^{(k)}(I)$ is independent of $\mathbf{V}^{k}=\sqrt{\frac{t P}{D_{n}}}\left[\mathbf{E}^{n}\right]_{1}^{k}$, and furthermore,

$\frac{1}{k} \mathbb{E}\left\|\mathbf{s}_{I}^{k}+\mathbf{V}^{k}\right\|^{2}=\frac{1}{k} \mathbb{E}\left[\left\|\mathbf{s}_{I}^{k}\right\|^{2}\right]+\frac{1}{k} \mathbb{E}\left[\left\|\mathbf{V}^{k}\right\|^{2}\right] \leq(1-t) P+t P$

so that the total input power constraint $P$ on the channel is met. By assumptions (i) and (ii) the actual channel noise $\mathbf{V}^{k}+$ $\mathbf{W}^{k}$ at the channel decoder can be regarded as an AWGN vector with per sample variance $t P+N$ which is independent of the channel encoder input. Under these assumptions the channel code has asymptotically vanishing error probability, i.e.,

$$
\lim _{n \rightarrow \infty} \operatorname{Pr}\{I \neq J\}=0 .
$$

It is well known that for the i.i.d. Gaussian source an asymptotically optimal source code can be chosen such that its codevectors lie on a sphere of radius $\sqrt{n\left(\sigma_{s}^{2}-D_{\tan }(N)\right)}$, i.e., we can assume $\frac{1}{n}\left\|\varphi_{d}^{(n)}(i)\right\|^{2}=\sigma_{s}^{2}-D_{\tan }(N)$ for all $i$. Using this fact and noting that (30) is equivalent to $\lim _{n \rightarrow \infty} \operatorname{Pr}\left\{\widetilde{\mathbf{X}}^{n} \neq \widehat{\widetilde{\mathbf{X}}}^{n}\right\}=0$, we obtain

$$
\lim _{n \rightarrow \infty} \frac{1}{n} \mathbb{E}\left\|\widetilde{\mathbf{X}}^{n}-\widehat{\widetilde{\mathbf{X}}}^{n}\right\|^{2}=0 .
$$

For simplicity we in fact assume that $\widetilde{\mathbf{X}}^{n}=\widehat{\widetilde{\mathbf{X}}}^{n}$ for large $n$. In this case, the average distortion can be written as

$$
\begin{aligned}
\frac{1}{n} \mathbb{E}\left\|\mathbf{X}^{n}-\widehat{\mathbf{X}}^{n}\right\|^{2} & =\frac{1}{n} \mathbb{E}\left\|\left(\widetilde{\mathbf{X}}^{n}+\mathbf{E}^{n}\right)-\left(\widehat{\widetilde{\mathbf{X}}}^{n}+\widehat{\mathbf{E}}^{n}\right)\right\|^{2} \\
& =\frac{1}{n} \mathbb{E}\left\|\mathbf{E}^{n}-\widehat{\mathbf{E}}^{n}\right\|^{2}
\end{aligned}
$$

On the other hand, from (28) we have

$$
\frac{1}{n} \mathbb{E}\left\|\mathbf{E}^{n}-\widehat{\mathbf{E}}^{n}\right\|^{2}=\frac{1}{n}\left\|\left[\mathbf{E}^{n}\right]_{1}^{k}-\frac{\sqrt{t P D_{n}}}{t P+N} \widehat{\mathbf{V}}^{k}\right\|^{2}+\frac{1}{n}\left\|\left[\mathbf{E}^{n}\right]_{k+1}^{n}\right\|^{2}
$$

where $\widehat{\mathbf{V}}^{k}=\mathbf{V}^{k}+\mathbf{W}^{k}+\mathbf{s}_{I}^{k}-\mathbf{s}_{J}^{k}$. It is well known that the channel codewords can be chosen to lie on a sphere of radius $\sqrt{k(1-t) P}$ (such an equi-energy codebook is often called a Gaussian codebook). Since (30) is equivalent to $\lim _{k \rightarrow \infty} \operatorname{Pr}\left\{\mathbf{s}_{I}^{k} \neq \mathbf{s}_{J}^{k}\right\}=0$, we obtain

$$
\lim _{k \rightarrow \infty} \frac{1}{k} \mathbb{E}\left\|\mathbf{s}_{I}^{k}-\mathbf{s}_{J}^{k}\right\|^{2}=0 .
$$

Again, for simplicity we actually assume $\mathbf{s}_{I}^{k}=\mathbf{s}_{J}^{k}$, so that $\widehat{\mathbf{V}}^{k}=\mathbf{V}^{k}+\mathbf{W}^{k}$ (for large $n$ ). Using (28), (33), and the assumption in (ii) that the components of $\mathbf{E}^{n}$ have equal variance $D_{n}$, we obtain ${ }^{2}$

$$
\begin{aligned}
\lim _{n \rightarrow \infty} \frac{1}{n} \mathbb{E}\left\|\mathbf{X}^{n}-\widehat{\mathbf{X}}^{n}\right\|^{2} \\
\quad=\lim _{n \rightarrow \infty} \frac{1}{n} \mathbb{E}\left\|\mathbf{E}^{n}-\widehat{\mathbf{E}}^{n}\right\|^{2} \\
\quad=\lim _{n \rightarrow \infty}\left(r \frac{D_{n}}{1+\frac{t P}{N}}+(1-r) D_{n}\right) \\
\quad=r \frac{D_{\tan }(N)}{1+\frac{t P}{N}}+(1-r) D_{\tan }(N)
\end{aligned}
$$

as desired. The preceding argument in fact forms the basis of a rigorous proof. The crucial point is to prove (30), i.e., the existence of a channel code of rate $R / r$ having vanishing error probability which also meets the total power constraint as in (29). Indeed, assuming (30) holds, we clearly have (31) and (34). It is then straightforward to show that (31) implies (35), and that (34) implies (36) as long as we have

$$
\lim _{n \rightarrow \infty} \frac{1}{k} \mathbb{E}\left\|\left[\mathbf{E}_{n}\right]_{1}^{k}\right\|^{2}=D_{\tan }(N) .
$$

It is easy to make sure (38) holds. Let $\ell$ be a positive integer which divides $n$ and assume the $n$-dimensional source code is the $n / \ell$-fold product of an $\ell$-dimensional vector quantizer $Q^{(\ell)}$ having rate $R$ (i.e., $Q^{(\ell)}$ is used $n / \ell$-times when encoding $\mathbf{X}^{n}$ ). If $\ell \rightarrow \infty$, then the rate-distortion performance (27) can be achieved by $Q^{(\ell)}$, and if in addition we have $\ell / n \rightarrow 0$, then (38) clearly holds.

Thus the entire proof hinges on the existence of channel codes with asymptotically vanishing error probability (30) under the power constraint $P$. In the remainder of the proof we show that such codes exist if one allows common randomization at the encoder and decoder. Common randomization, already used in the context of both source and channel coding (see, e.g., [34], [7], [8] and [3]), ensures that the total input power meets the power constraint and also makes the transmitted channel codeword and the "noise" $\mathbf{V}^{k}+\mathbf{W}^{k}$ independent. In what follows we first show that the average channel noise $\frac{1}{k}\left\|\mathbf{V}^{k}+\mathbf{W}^{k}\right\|$ is concentrated near its expectation $t P+N$ with large probability, and then use this fact in showing that the desired channel code exists.

Recall that $D_{\tan }(N)=\sigma_{s}^{2} 2^{-2 R}$ is the distortion-rate function at rate $R$ of a memoryless Gaussian source with variance $\sigma_{s}^{2}$. It is known (see., e.g., [24] or [15]) that one can choose $Q^{(\ell)}$ so that its codevectors lie on a sphere of radius $\sqrt{\ell\left(\sigma_{s}^{2}-D_{\tan }(N)\right)}$ and it has asymptotically optimal distortion $\lim _{\ell \rightarrow \infty} \frac{1}{\ell} \mathbb{E}\left\|\mathbf{X}^{\ell}-Q^{(\ell)}\left(\mathbf{X}^{\ell}\right)\right\|^{2}=D_{\text {tan }}(N)$, which implies (27) since $D_{n}=\frac{1}{\ell} \mathbb{E} \mid \mathbf{X}^{\ell}-Q^{(\ell)}\left(\mathbf{X}^{\ell}\right) \|^{2}$ by the source code construction.

Since $\left[\mathbf{E}^{n}\right]_{1}^{k}$ is the concatenation of $m^{\prime}=k / \ell$ independent copies of $\mathbf{X}^{\ell}-Q^{(\ell)}\left(\mathbf{X}^{\ell}\right)$, and $\mathbf{V}^{k}=\sqrt{\frac{t P}{D_{n}}}\left[\mathbf{E}^{n}\right]_{1}^{k}$, we have that $\left\|\mathbf{V}^{k}\right\|^{2}$ is the sum of $m^{\prime}=k / \ell$ independent random variables with mean $\frac{t P}{D_{n}} \mathbb{E}\left\|\mathbf{X}^{\ell}-Q^{(\ell)}\left(\mathbf{X}^{\ell}\right)\right\|^{2}=\ell t P$. Thus if $\ell$ is fixed,

\footnotetext{
${ }^{2}$ With these assumptions, $\frac{\sqrt{t P D_{n}}}{t P+N} \widehat{\mathbf{V}}^{k}$ becomes the MMSE estimate of $\left[\mathbf{E}^{n}\right]_{1}^{k}$.
} 
the weak law of large numbers implies

$$
\lim _{k \rightarrow \infty} \operatorname{Pr}\left\{\left|\frac{1}{k}\left\|\mathbf{V}^{k}\right\|^{2}-t P\right|>\epsilon\right\}=0
$$

for all $\epsilon>0$. Clearly, we can choose an $\ell$ sequence such that $\ell \rightarrow \infty, \ell / k=\ell /(r n) \rightarrow 0$ and (39) still holds. For the rest of the proof we assume that $\ell$ increases with $n$ (and $k$ ) in this fashion. We have $\frac{1}{k}\left\|\mathbf{V}^{k}+\mathbf{W}^{k}\right\|^{2}=$ $\frac{1}{k}\left\|\mathbf{V}^{k}\right\|^{2}+\frac{1}{k}\left\|\mathbf{W}^{k}\right\|^{2}+\frac{2}{k}\left(\mathbf{V}^{k}\right)^{T} \mathbf{W}^{k}$, where $\frac{1}{k}\left\|\mathbf{W}^{k}\right\|^{2}$, being the average of $k$ i.i.d. random variables of mean $N$, converges to $N$ in probability as $k \rightarrow \infty$. A direct calculation shows that $\mathbb{E}\left[\left(\frac{1}{k}\left(\mathbf{V}^{k}\right)^{T} \mathbf{W}^{k}\right)^{2}\right]=\frac{N}{k^{2}} \mathbb{E}\left\|\mathbf{V}^{k}\right\|^{2}=\frac{N}{k} t P$, which converges to zero as $k \rightarrow \infty$, implying through Chebyshev's inequality that $\operatorname{Pr}\left\{\left|\frac{2}{k}\left(\mathbf{V}^{k}\right)^{T} \mathbf{W}^{k}\right|>\epsilon\right\} \rightarrow 0$ as $k \rightarrow \infty$ for all $\epsilon>0$. Combining these facts with (39) we obtain that for all $\epsilon>0$,

$$
\lim _{k \rightarrow \infty} \operatorname{Pr}\left\{\left|\frac{1}{k}\left\|\mathbf{V}^{k}+\mathbf{W}^{k}\right\|^{2}-(t P+N)\right|>\epsilon\right\}=0 .
$$

Now consider the fictitious $k$-dimensional vector channel with input power constraint $k(1-t) P$ and additive noise which is independent of the input and has the same distribution as $\mathbf{V}^{k}+\mathbf{W}^{k}$. The key point is that (40) allows us to use Theorem 1 in [14] which, when applied to our setup, states that given an additive noise channel with power constraint $k(1-t) P$ and input-independent, possibly non-ergodic noise which satisfies (40), there exists a sequence of channel codes $\left(\psi_{e}^{(k)}, \psi_{d}^{(k)}\right)$ which has rate $\frac{1}{2} \log \left(1+\frac{(1-t) P}{t P+N}\right)$ and equienergy (Gaussian) codebook and whose error probability on this channel approaches zero as $k \rightarrow \infty$. (Thus, in effect, a channel code designed for the worst case AWGN noise also works for non-Gaussian channel noise of equal power.)

We will use common randomization to apply $\left(\psi_{e}^{(k)}, \psi_{d}^{(k)}\right)$ to the real system where $\mathbf{V}^{k}+\mathbf{W}^{k}$ is not independent of the channel input. Let $\Pi$ denote a random permutation of the indices $1, \ldots, 2^{n R}$ which is uniformly drawn from the set of all $\left(2^{n R}\right)$ ! permutations and is independent of the source $\mathbf{X}^{n}$ and the channel noise $\mathbf{W}^{k}$. Assume that $\Pi$ is know at both the encoder and the decoder. At the encoder apply $\Pi$ to the output index $I$ of the source encoder before channel coding, so that the input to the channel encoder is $\Pi(I)$. At the decoder side, if $J$ is the output index at the channel decoder, then $\Pi^{-1}(J)$ is sent to the source decoder, where $\Pi^{-1}$ denotes the inverse of $\Pi$. It is easy to see that the channel with input $I$ and output $\Pi^{-1}(J)$ is statistically equivalent to the discrete channel realized when $\left(\psi_{e}^{(k)}, \psi_{d}^{(k)}\right)$ is used on the fictitious channel with a uniform distribution on its input index set. Since $\left(\psi_{e}^{(k)}, \psi_{d}^{(k)}\right)$ has asymptotically vanishing error probability on the fictitious channel, for the real system we also have $\lim _{k \rightarrow \infty} \operatorname{Pr}\left\{I \neq \Pi^{-1}(J)\right\}=0$. It remains to show that the total power input power on the channel does not exceed $P$. Since $\mathbf{s}_{\Pi(I)}^{k}=\psi_{e}^{(k)}(\Pi(I))$ is independent of $\mathbf{V}^{k}$,

$$
\begin{aligned}
& \frac{1}{k} \mathbb{E}\left\|\mathbf{s}_{\Pi(I)}^{k}+\mathbf{V}^{k}\right\|^{2} \\
& \quad=\frac{1}{k} \mathbb{E}\left\|\mathbf{s}_{\Pi(I)}^{k}\right\|^{2}+\frac{1}{k} \mathbb{E}\left\|\mathbf{V}^{k}\right\|^{2}+\frac{2}{k} \mathbb{E}\left[\mathbf{s}_{\Pi(I)}^{k}\right]^{T} \mathbb{E}\left[\mathbf{V}^{k}\right](4)
\end{aligned}
$$

where $\frac{1}{k} \mathbb{E}\left\|\mathbf{s}_{\Pi(I)}^{k}\right\|^{2}=(1-t) P$ and $\frac{1}{k} \mathbb{E}\left\|\mathbf{V}^{k}\right\|^{2}=t P$. Let $\mathbf{m}^{\ell} \triangleq \mathbb{E}\left[\mathbf{X}^{\ell}-Q^{(\ell)}\left(\mathbf{X}^{\ell}\right)\right]$. Then

$$
\begin{aligned}
D_{n} & =\frac{1}{n} \mathbb{E}\left\|\mathbf{X}^{\ell}-Q^{(\ell)}\left(\mathbf{X}^{\ell}\right)\right\|^{2} \\
& =\frac{1}{\ell} \mathbb{E}\left\|\mathbf{X}^{\ell}-Q^{(\ell)}\left(\mathbf{X}^{\ell}\right)-\mathbf{m}^{\ell}\right\|^{2}+\frac{1}{\ell}\left\|\mathbf{m}^{\ell}\right\|^{2} \\
& \geq D_{\tan }(N)+\frac{1}{\ell}\left\|\mathbf{m}^{\ell}\right\|^{2}
\end{aligned}
$$

where the inequality holds since $Q^{(\ell)}\left(\mathbf{X}^{\ell}\right)+\mathbf{m}^{\ell}$ is a rate $R$ quantizer for $\mathbf{X}^{\ell}$. This implies $\lim _{\ell \rightarrow \infty} \frac{1}{\ell}\left\|\mathbf{m}^{\ell}\right\|^{2}=0$. Since $\frac{1}{\ell}\left\|\mathbf{m}^{\ell}\right\|^{2} \frac{t P}{D_{n}}=\frac{1}{k}\left\|\mathbb{E}\left[\mathbf{V}^{k}\right]\right\|^{2}$, applying Cauchy-Schwarz inequality yields $\lim _{k \rightarrow \infty} \frac{1}{k} \mathbb{E}\left[\mathbf{s}_{\Pi(I)}^{k}\right]^{T} \mathbb{E}\left[\mathbf{V}^{k}\right]=0$. Substituting this into (41) shows that $\lim _{k \rightarrow \infty} \frac{1}{k} \mathbb{E}\left\|\mathbf{s}_{\Pi(I)}^{k}+\mathbf{V}^{k}\right\|^{2}=$ $(1-t) P+t P$; thus, the power constraint is (asymptotically) satisfied.

\section{ACKNOWLEDGMENT}

The authors would like to sincerely thank Ram Zamir for suggesting the common randomization idea in the proof of Proposition 1, and the reviewers for very helpful comments. Particular thanks are due to one reviewer for insightfully suggesting the study of the HDA* system and pointing out that this system can achieve OPTA.

\section{REFERENCES}

[1] T. Berger, Rate Distortion Theory: A Mathematical Basis for Data Compression. Upper Saddle River, NJ: Prentice-Hall, 1971.

[2] G. Caire and K. Narayanan, "On the distortion SNR exponent of hybrid digital-analog space-time coding," IEEE Trans. Inform. Theory, vol. 53, pp. 2867-2878, Aug. 2007.

[3] J. Chen, C. Tian, T. Berger, and S. S. Hemami, " Multiple description quantization via Gram-Schmidt orthogonalization," IEEE Trans. Inform. Theory, vol. 52, no. 12, pp. 5197-5217, Dec. 2006.

[4] T. M. Cover and J. A. Thomas, Elements of Information Theory, 2nd ed. New York: Wiley, 2006.

[5] H. Coward, "Joint source-channel coding: development of methods and utilization in image communications," Ph.D thesis, Norwegian University of Science and Engineering (NTNU), 2001.

[6] H. Coward and T. A. Ramstad, "Robust image communication using bandwidth reducing and expanding mappings," in Proc. 34th Asilomar Conf., Oct.-Nov. 2000, pp. 1384-1388.

[7] U. Erez, S. Shamai, and R. Zamir, "Capacity and lattice strategies for canceling known interference," IEEE Trans. Inform. Theory, vol. 51, no. 11 , pp. 3820-3833, Nov. 2005.

[8] U. Erez and R. Zamir, "Achieving $\frac{1}{2} \log (1+$ SNR) on the AWGN channel with lattice encoding and decoding," IEEE Trans. Inform. Theory, vol. 50, no. 10, pp. 2293-2314, Oct. 2004.

[9] N. Farvardin and V. Vaishampayan, "On the performance and complexity of channel-optimized vector quantizers," IEEE Trans. Inform. Theory, vol. 37, pp. 155-159, Jan. 1991.

[10] A. Fuldseth and T. A. Ramstad, "Bandwidth compression for continuous amplitude channels based on vector approximation to a continuous subset of the source signal space," in Proc. IEEE ICASSP, Apr. 1997, pp. 3093-3096.

[11] F. Hekland, G. E. Øien, and T. A. Ramstad, "Using 2:1 Shannon mapping for joint source-channel coding," in Proc. IEEE Data Compression Conf., Mar. 2005, pp. 223-232.

[12] T. T. Kim, M. Skoglund, and G. Caire, "Distortion exponents over fading MIMO channels with quantized feedback," in Proc. IEEE ISIT, June 2007, pp. 2236-2240.

[13] I. Kozintsev and K. Ramchandran, "Hybrid compressed-uncompressed framework for wireless image transmission," in Proc. IEEE Int. Conf. Commun., June 1997, pp. 77-80.

[14] A. Lapidoth, "Nearest neighbor decoding for additive non-Gaussian noise channel," IEEE Trans. Inform. Theory, vol. 42, no. 5, pp. 15201529, Sept. 1996.

[15] A. Lapidoth, "On the role of mismatch in rate distortion theory," IEEE Trans. Inform. Theory, vol. 43, pp. 38-47, Sept. 1997. 
[16] J. M. Lervik, A. Grovlen, and T. A. Ramstad, "Robust digital signal compression and modulation exploiting the advantages of analog communications," in Proc. IEEE GLOBECOM, Nov. 1995, pp. 1044-1048.

[17] J. Lim and D. L. Neuhoff, "Joint and tandem source-channel coding with complexity and delay constraints," IEEE Trans. Commun., vol. 51, pp. 757-766, May 2003.

[18] Y. Linde, A. Buzo, and R. M. Gray, "An algorithm for vector quantizer design," IEEE Trans. Commun., vol. 28, pp. 84-95, Dec. 1980.

[19] U. Mittal and N. Phamdo, "Hybrid digital-analog (HDA) joint sourcechannel codes for broadcasting and robust communications," IEEE Trans. Inform. Theory, vol. 48, pp. 1082-1102, May 2002.

[20] H. C. Papadopoulos and C. E. W. Sundberg, "Simultaneous broadcasting of analog FM and digital audio signals by means of adaptive precanceling techniques," IEEE Trans. Commun., vol. 46, pp. 12331242, Sept. 1998.

[21] N. Phamdo and F. Alajaji, "Soft-decision demodulation design for COVQ over white, colored, and ISI Gaussian channels," IEEE Trans. Commun., vol. 48, no. 9, pp. 1499-1506, Sept. 2000.

[22] N. Phamdo and U. Mittal, "A joint source-channel speech coder using hybrid digital-analog (HDA) modulation," IEEE Trans. Speech and Audio Processing, vol. 10, no. 4, pp. 222-231, May 2002.

[23] Z. Reznic, M. Feder, and R. Zamir, "Distortion bounds for broadcasting with bandwidth expansion," IEEE Trans. Inform. Theory, vol. 52, no. 8, pp. 3778-3788, Aug. 2006.

[24] D. Sakrison, "A geometric treatment of the source encoding of a Gaussian random variable," IEEE Trans. Inform. Theory, vol. 14, no. 3, pp. 481-486, May 1968.

[25] S. Sesia, G. Caire, and G. Vivier, "Lossy transmission over slow-fading AWGN channels: a comparison of progressive, superposition and hybrid approaches," in Proc. IEEE ISIT'05, Sept. 2005, pp. 224-228.

[26] S. Shamai, S. Verdú, and R. Zamir, "Systematic lossy source/channel coding," IEEE Trans. Inform. Theory, vol. 44, no. 2, pp. 564-579, Mar. 1998.

[27] C. E. Shannon, "A mathematical theory of communication," Bell Syst. Tech. J., vol. 27, pp. 379-423; 623-656, 1948.

[28] M. Skoglund, N. Phamdo, and F. Alajaji, "Design and performance of VQ-based hybrid digital-analog joint source-channel codes," IEEE Trans. Inform. Theory, vol. 48, no. 3, pp. 708-720, Mar. 2002.

[29] M. Skoglund, N. Phamdo, and F. Alajaji, "Hybrid digital-analog sourcechannel coding for bandwidth compression/expansion," IEEE Trans. Inform. Theory, vol. 52, no. 8, pp. 3757-3763, Aug. 2006.

[30] N. Tanabe and N. Farvardin, "Subband image coding using entropycoded quantization over noisy channels," IEEE J. Select. Areas Commun., vol. 10, pp. 926-943, June 1992.

[31] V. Vaishampayan, "Combined source-channel coding for bandlimited waveform channels," Ph.D. thesis, University of Maryland, College Park, USA, 1989.

[32] V. Vaishampayan and S. I. R. Costa, "Curves on a sphere, shift-map dynamics, and error control for continuous alphabet sources," IEEE Trans. Inform. Theory, vol. 49, no. 7, pp. 1658-1672, July 2003.

[33] Y. Wang, "Hybrid digital-analog source-channel coding and information hiding: Information-theoretic perspectives," Ph.D. thesis, Queen's University, Kingston, ON, Canada, 2007.

[34] R. Zamir and M. Feder, "On universal quantization by randomized uniform/lattice quantizers," IEEE Trans. Inform. Theory, vol. 38, no. 2, pp. 428-436, Mar. 1992.

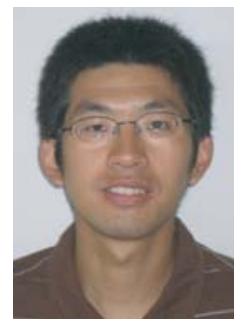

Yadong Wang was born in Jiujiang, JiangXi, China, in 1977. He received the B.S. and M.S. degrees in computational mathematics from Jilin University, Changchun, China, and the Ph.D degree in applied mathematics from Queen's University, Kingston, ON, Canada, in 1999, 2002, and 2007, respectively. He was with the Communications and Signal Processing Lab, Queen's University. His research interests include information theory, joint sourcechannel coding, information hiding, and digital watermarking. He now works at the BMO Financial Group, Toronto, ON, Canada as a senior business analyst.

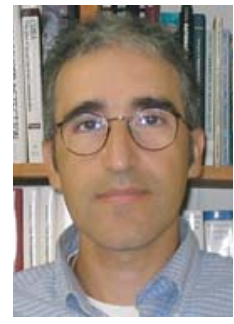

Fady Alajaji (S'90-M'94-SM'00) was born in Beirut, Lebanon, on May 1, 1966. He received the B.E. degree with distinction from the American University of Beirut, Lebanon, and the M.Sc. and Ph.D. degrees from the University of Maryland, College Park, all in electrical engineering, in 1988, 1990 and 1994, respectively. He held a postdoctoral appointment in 1994 at the Institute for Systems Research, University of Maryland.

In 1995, he joined the Department of Mathematics and Statistics at Queen's University, Kingston, Ontario, where he is currently a Professor of Mathematics and Engineering. Since 1997, he has also been cross-appointed in the Department of Electrical and Computer Engineering at the same university. His research interests include information theory, joint source-channel coding, error control coding, data compression and digital communications.

Dr. Alajaji currently serves as Editor for Source and Source-Channel Coding for the IEEE TRANSACTIONS ON COMMUNICATIONS. He served as cochair of the 1999 Canadian Workshop on Information Theory, as co-chair of the Technical Program Committee (TPC) of the 2004 Biennial Symposium on Communications, and as a TPC member of several international conferences and workshops. He received the Premier's Research Excellence Award from the Province of Ontario.

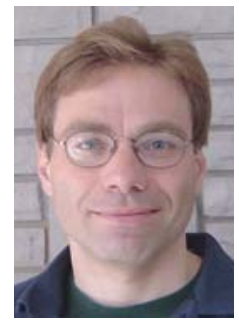

Tamas Linder (S'92-M'93-SM'00) was born in Budapest, Hungary, in 1964. He received the M.S. degree in electrical engineering from the Technical University of Budapest in 1988, and the Ph.D degree in electrical engineering from the Hungarian Academy of Sciences in 1992.

He was a post-doctoral researcher at the University of Hawaii in 1992 and a Visiting Fulbright Scholar at the Coordinated Science Laboratory, University of Illinois at Urbana-Champaign during 1993-1994. From 1994 to 1998 he was a faculty member in the Department of Computer Science and Information Theory at the Technical University of Budapest. From 1996 to 1998 he was also a visiting research scholar in the Department of Electrical and Computer Engineering, University of California, San Diego. In 1998 he joined Queen's University where he is now a Professor of Mathematics and Engineering in the Department of Mathematics and Statistics. His research interests include communications and information theory, source coding and vector quantization, machine learning, and statistical pattern recognition.

Dr. Linder received the Premier's Research Excellence Award of the Province of Ontario in 2002 and the Chancellor's Research Award of Queen's University in 2003. He was an Associate Editor for Source Coding of the IEEE TRANSACTIONS ON INFORMATION THEORY in 2003-2004. 